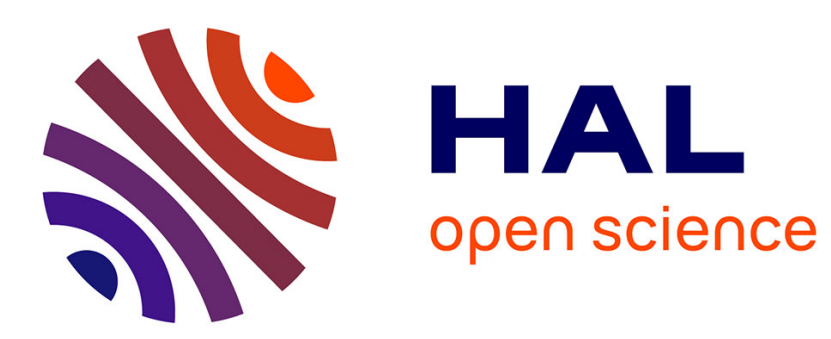

\title{
Simulation of a Scramjet Combustor: A Priori Study of Thermochemistry Tabulation Techniques
}

Pascale Domingo, Jiangheng Loïc Ruan, Lisa Bouheraoua, Guillaume Ribert

\section{To cite this version:}

Pascale Domingo, Jiangheng Loïc Ruan, Lisa Bouheraoua, Guillaume Ribert. Simulation of a Scramjet Combustor: A Priori Study of Thermochemistry Tabulation Techniques. Flow, Turbulence and Combustion, 2021, 106, pp.1241 - 1276. 10.1007/s10494-020-00184-4 . hal-03041431

HAL Id: hal-03041431

https://hal-normandie-univ.archives-ouvertes.fr/hal-03041431

Submitted on 4 Dec 2020

HAL is a multi-disciplinary open access archive for the deposit and dissemination of scientific research documents, whether they are published or not. The documents may come from teaching and research institutions in France or abroad, or from public or private research centers.
L'archive ouverte pluridisciplinaire HAL, est destinée au dépôt et à la diffusion de documents scientifiques de niveau recherche, publiés ou non, émanant des établissements d'enseignement et de recherche français ou étrangers, des laboratoires publics ou privés. 


\title{
Simulation of a Scramjet Combustor: A Priori Study of Thermochemistry Tabulation Techniques
}

\author{
J.L. Ruan • L. Bouheraoua • \\ P. Domingo • G. Ribert
}

Received: date / Accepted: date

\begin{abstract}
Large eddy simulations (LES) of a scramjet combustor are reported in this paper. The case under study is a cavity-based combustion chamber that is experimentally studied at the U.S Air Force Research Laboratory. The chamber is fed by eleven injectors. The computational domains are either simplified including only one or two injectors or complete with the 11 injectors. A good agreement is found between experimental data (velocities measured by PIV) and results from the LES if the kinetic used is chosen with care. A high temperature is found inside the cavity promoting a reactive zone located in the mixing layer where the flow velocity is high. At this location, the combustion occurs first in a diffusion dominated regime followed by the efficient burning of a well mixed mixture (rich then lean). A significant diffusion dominated burning is also found inside the cavity, mostly at the interface between the two recirculation zones. The simulation of the complete geometry revealed a transverse phenomenon on the temperature and mixing fields, but which had nevertheless little effect on the comparison with the available experimental data.

A tabulation of the chemistry based on a premixed flamelet library without compressibility effects has been tested a priori on the results of the simulation with one injector. Good results on temperature and $\mathrm{H}_{2} \mathrm{O}$ fields are found. Significant localized discrepancies appeared on $\mathrm{CO}$ and $\mathrm{CO}_{2}$ fields due to the complexity of the combustion regimes, while compressibility effects were found to be weak for the configuration studied.
\end{abstract}

Keywords Supersonic combustion · Large-eddy simulation · Cavity-based scramjet · Premixed Flamelet Tabulation

\footnotetext{
J.L. Ruan · L. Bouheraoua · P. Domingo · G. Ribert

Corresponding author: Guillaume Ribert

E-mail: guillaume.ribert@insa-rouen.fr

CORIA-CNRS, Normandie Université, INSA de Rouen Normandie

76000 Rouen, France
} 


\section{Introduction}

Despite the increase in high-performance computing, it remains challenging to simulate complete combustion chambers while ensuring accurate numerical methods, reliable description of the thermochemistry and efficient models for the phenomena unresolved by the mesh. In particular, it should be noted that few turbulent combustion models are specifically developed for the supersonic flow regime and the classical subsonic modeling techniques are very often transposed to the supersonic case [4]. This is the case for tabulation techniques, that allow for drastically reducing the number of transported scalars $[29,31]$. Indeed, a look-up table contains all information about the chemical system from a well-chosen collection of canonical flames or processes. Thermodynamical variables are then tabulated as functions of a small set of control variables that are transported with the flow [7]. However, dealing with tabulated thermochemistry in a highly compressible mixture constitutes a challenge $[35,36]$, as the compressibility effects are not generally accounted for in table constructions. For instance, let's consider the supersonic burner of Cheng [6] in which a sonic hydrogen fuel jet at $545 \mathrm{~K}$ interacts with an annular jet of hot $(1250 \mathrm{~K})$ and vitiated air at Mach 2 . Both flows exit into a quiescent atmosphere creating shocks as shown in Fig. 1 through the pressure field and the scatter plot of temperature, before combustion starts. The combination of

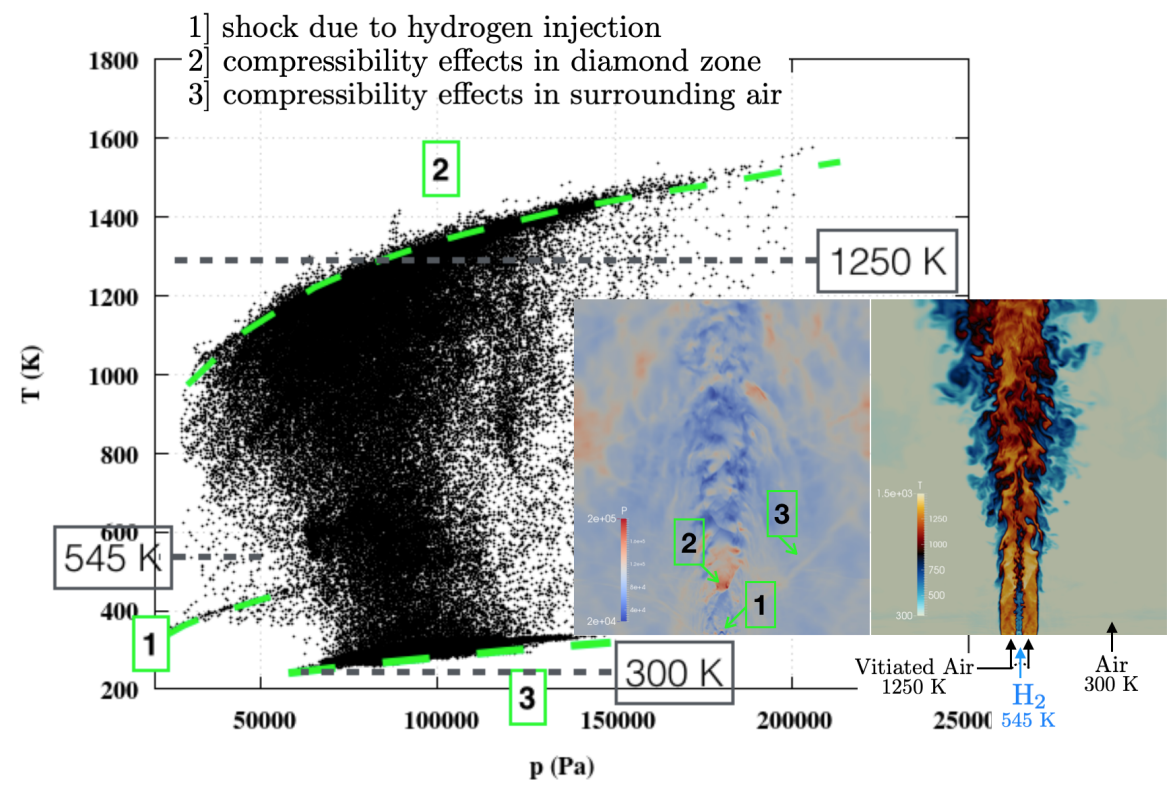

Fig. 1 Scatter plot of temperature versus pressure for an instantaneous non reactive solution [4] of Cheng's burner. The compressible effects on the mixing temperature are highlighted by green dashed lines. In the insert on the right: pressure (left) and temperature (right) fields. 
hydrogen, oxidizer and hot temperature causes autoignition phenomena that occur at different locations in the jet and result in different combustion regimes as well as the birth of detonations [4]. Building a look-up table thus becomes legitimate. Perfectly stirred reactor (PSR) have been computed and all thermochemistry infirmations (temperature, species mass fractions, etc.) are stored function of the progress variable for various values of the mixing and pressure levels between 20 and $220 \mathrm{KPa}$. However, using the inlet temperature of the experiment as an input data of the table $\left(T_{0}^{\text {mix }}\right)$, i.e. using Table 1 in Fig. 2, leads to incorrect predictions. Indeed, compressibility effects must be consid-
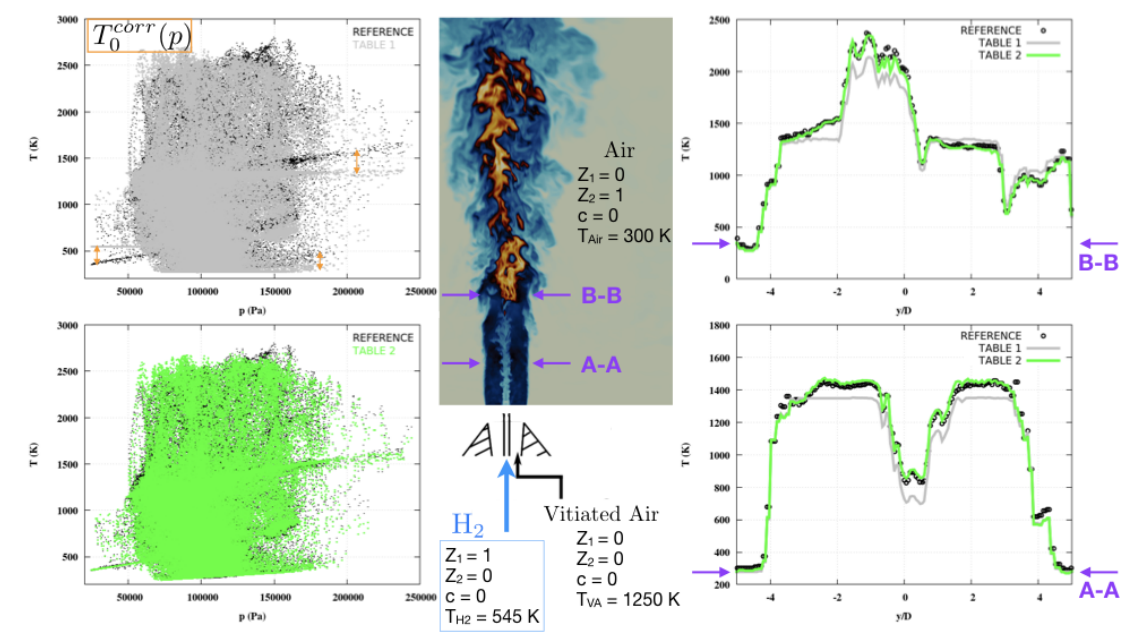

$\begin{array}{ll}\text { TABLE 1: } & T_{0}=T_{0}^{\text {mix }}\left(Z_{1}, Z_{2}\right) \\ \text { TABLE 2: } & T_{0}=T_{0}^{\text {shock }}\left(Z_{1}, Z_{2}, p\right)=T_{0}^{\text {mix }}\left(Z_{1}, Z_{2}\right)+T_{0}^{\text {corr }}(p) \\ & T_{0}^{\text {mix }}=T_{H_{2}} Z_{1}+T_{V A}\left(1-Z_{1}\right)\left(1-Z_{2}\right)+T_{A i r} Z_{2}\end{array}$

Fig. 2 Compressibility effects on look-up table (TABLE 1 vs. TABLE 2). Comparison of temperature versus pressure scatter plots for the reactive case (left) and at two downstream locations (right) along the jet axis: $x / D=10.8[\mathrm{~A}-\mathrm{A}]$ and $x / D=21.5[\mathrm{~B}-\mathrm{B}]$, with $D=$ $2.36 \mathrm{~mm} . Z_{1}$ describes the mixing between hydrogen and vitiated air, and $Z_{2}$ the mixing between pure air and vitiated air. The value of temperature to enter the look-up table is $T_{0}$. The correction $\left(T_{0}^{\text {corr }}\right)$ applied to TABLE 2 to account for compressibility effects is simply defined as the difference of the grey and green curves of Fig. 1

ered when building up the look-up table. In Cheng's burner, these effects come from the shock arrangements which appear before the combustion zone and which modify the values of thermodynamical variables such as temperature. This temperature modification can be deduced from Fig. 1 by the difference between the temperature from the green dash lines and the temperature from the grey dash lines $\left(T_{0}^{\text {corr }}\right)$. Using $T_{0}^{\text {shock }}\left(=T_{0}^{\text {mix }}+T_{0}^{\text {corr }}\right)$ for the creation of the look-up table (Table 2) instead of $T_{0}^{\text {mix }}$ leads to a better description of the 
temperature field as shown either on the scatter plot of temperature (left) or on a cut of temperature at two different locations downstream the burner exit (right), in Fig. 2. In the case of hydrogen fuel, the gain in CPU time is small because the CPU cost of the chemical kinetic is not prohibitive. However, it becomes significant for hydrocarbons. Note that another advantage of chemistry tabulation consists in the easier way to introduce combustion subgrid modeling through presumed probability density functions.

Similar questions are now addressed: is the use of a tabulation method for thermochemistry feasible in the case of a cavity-based scramjet configuration? Will the compressibility effects play also an important role in that case? In such a device, air flows at a supersonic velocity over a cavity that traps hot products coming from the decomposition of fuel and air by means of a primary vortex positioned inside the cavity. The reaction zone is located between the air flow and the cavity, and a secondary vortex acts as a fuel pool mixed with hot burned products in a dead zone at the front of the cavity [34]. Again, the effects of compressibility can play a major role in the creation of a look-up table. This difficulty is exacerbated by the wide variety of flame regimes that can be encountered: premixed, non-premixed, partially-premixed, or near (or in) the distributed-reaction-like regime [13], i.e. when high-speed reacting flows have also high levels of turbulent fluctuations.

To tackle this issue, a high-fidelity simulation of a scramjet combustor has been engaged. In the facility from AFRL [37], the air flow is accelerated through a nozzle to reach supersonic speed and enters the isolator at nominal Mach 2, with a total temperature and pressure of $589 \mathrm{~K}$ and $483 \mathrm{kPa}$, respectively. The air flow then meets the fuel (ethylene) inside the cavity where combustion has been shown to take place. The back of the cavity has an angle to limit streamwise pressure oscillations. An array of 11 fuel injectors are evenly distributed on the aft cavity wall. This experiment has already been studied by LES (Large-Eddy Simulations) or RANS (Reynolds Average Navier-Stokes) $[2,16,17,34]$. Particle image velocimetry are available inside the cavity [37]. In the present study, a high-fidelity simulation of the AFRL test-bench is performed with a focus on the impact of the number of injectors. Indeed, simulating a single injector with periodic conditions on the sides can be enough to highlight the turbulence-flame interaction acting inside the cavity. However, to really encompass this interaction in detail, that is to say with transverse phenomena, it is necessary to simulate the complete configuration, which will necessarily entail a huge CPU cost. The choice of a combustion modeling will then be crucial and it is proposed, in this study, to validate a priori the use of a tabulated approach. 


\section{LES of scramjet combustor}

\subsection{Experimental and numerical set-up}

Experiments conducted at the US Air Force Research Laboratory (AFRL) by Tuttle et al. [37] will then support the goal described above. Figure 3 is a summary of the experimental configuration and more details can be found in [37] or in numerical articles $[2,16,17,34]$. The case under study has a fuel flow

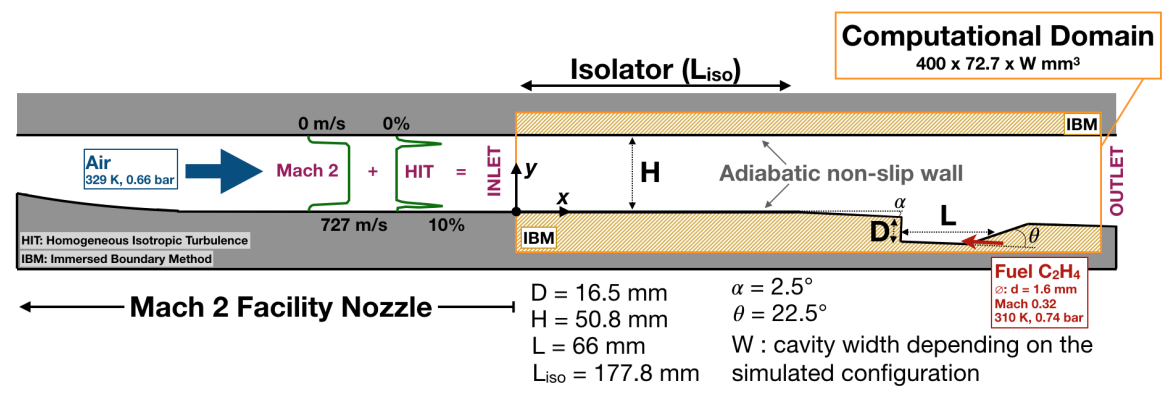

Fig. 3 Sketch of the AFRL supersonic wind tunnel.

rate of 99 Standard Liters Per Minute.

The CORIA in-house code, SiTCom-B, is used for the computations $[3,10,15]$. SiTCom-B is a fully compressible structured code, based on an explicit Finite Volumes scheme, and has already shown its capability to predict supersonic flows and combustion $[4,14,34]$.

In the present simulations, different options are considered: in Tab. 1, RCA1 corresponds to the simulation of a single injector centered at the middle plane of the computational domain, RCA2 has two injectors and RCA11 is for the complete geometry of the cavity including the 11 injectors. The two injectors case RCA2 $(z \in[-12.7 \mathrm{~mm}, 12.7 \mathrm{~mm}])$ has been initialized with the one injector solution RCA1 $(z \in[-12.7 \mathrm{~mm}, 0])$ by symmetry to the axis $z=0$. The center of each injector is at $z=-6.35 \mathrm{~mm}$ and $z=6.35 \mathrm{~mm}$, respectively. The boundaries at $z=-12.7 \mathrm{~mm}$ and $z=12.7 \mathrm{~mm}$ are prescribed as periodic. The mesh is composed of 90 millions of cells and needs approximately 200000 hours CPU time to gather statistics of $4 \mathrm{~ms}$ physical time. The eleven injectors case RCA11 $(z \in[-82.55 \mathrm{~mm}, 69.85 \mathrm{~mm}])$ has also been initialized from the one injector solution by duplicating the solution for each injector, located at $z=-69.85,-57.15,-44.45,-31.75,-19.05,-6.35,6.35,19.05$, $31.75,44.45,57.15 \mathrm{~mm}$ respectively. Note that the width of the case RCA11 is $152.4 \mathrm{~mm}$ which is equal to $12 \times 12.7 \mathrm{~mm}$ and not 11 . Actually, the distance between the first/last injector and the wall is 1.5 times higher than the distance between two injectors: $19.05 \mathrm{~mm}$ instead of $12.7 \mathrm{~mm}$. The centerplane of the whole geometry is the centerplane of the 6 -th injector which is located at $z=-6.35 \mathrm{~mm}$. The side walls are modeled with adiabatic non-slipping wall 
conditions. The mesh of the complete domain has 542 millions of cells and requires about 3000000 hours CPU time for $4 \mathrm{~ms}$ of statistics. The airflow is injected with homogeneous isotropic turbulence with an intensity of $10 \%$ of the local speed. Each injector has the same mass flow rate, which is $1 / 11 \times 99$ SLPM, leading to a velocity of $115 \mathrm{~m} / \mathrm{s}$ per injector. Two mesh resolution have

\begin{tabular}{c|ccc}
\hline \hline Case & Cavity width & Injector center coordinate & Side BC \\
\hline RCA1 & 12.7 & $z=z_{0}$ & Periodic \\
RCA2 & 25.4 & $z= \pm z_{0}$ & Periodic \\
RCA11 & 152.4 & $z_{i}=z_{0}+i \times 12.7$ & Adiabatic non-slip wall \\
\hline \hline
\end{tabular}

Table 1 Parameters of reactive cases with different number of injectors in $\mathrm{mm} . z_{0}=$ $6.35 \mathrm{~mm}$ and $i \in[-5,+5]$. BC: boundary conditions. Coarse mesh.

been used for the simulations with one injector, a coarse one with a resolution around $200 \mu \mathrm{m}$ (RCA1) and a more refined one with a resolution around $100 \mu \mathrm{m}$ (RFA1) in the reactive zones inside and above the cavity. For the simulations with two or eleven injectors, only the coarse resolution has been used.

\subsection{Chemistry of ethylene}

The reduced kinetic scheme used in this study has 21 reacting species plus nitrogen $[21]$ and is named S22. It has been reduced from a detailed mechanism based on the mechanism called USC-Mech II from the University of South California, composed of 75 species and 529 elementary reactions. S22 has been compared with success to its seminal detailed mechanism for auto-ignition, extinction in Perfectly-Stirred Reactor (PSR) and premixed laminar flames for a large range of initial conditions [21]: pressure $P \in[1-50]$ bar, temperature $T \in[1000-1800] \mathrm{K}$ and equivalence ratio $\phi \in[0.5-1.5]$.

Autoignition computations for lean and rich mixtures have been performed with S22 at the pressure of the air inlet, $P=0.66$ bar, but varying the inlet temperature. In Fig. 4 (left), the ignition delay decreases when increasing the inlet temperature but also when the equivalence ratio increases. As a consequence, a rich mixture would burn faster than a lean one. Kopp et al. [18] have found that under high-pressure conditions (around 20 bar), the richer the mixture is, the faster it reacts. However, under low-pressure conditions (around 1 bar) the leaner the mixture is, the faster it ignites. Fig. 4 (right) shows a comparison between the numerical results from S22 and the experimental data from Kopp et al. [18] at low pressure, $P=1.1$ bar. The simulations and experiments show strong disparities for $\phi=0.5$ and $\phi=1$. Kopp et al. also propose a detailed mechanism Aramco 1.3 [25] which could overcome the previous defect of S22. But such mechanism is composed of 346 species 

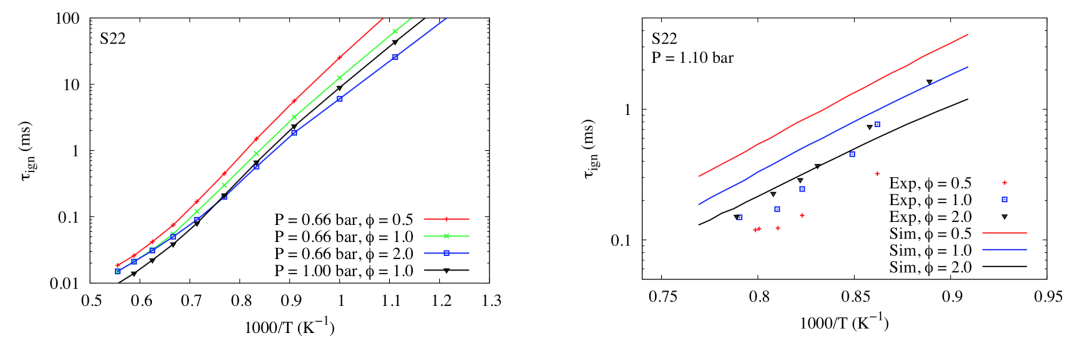

Fig. 4 Comparaison of ignition delay for S22 at several initial conditions.

and thus does not fit a 3D large eddy simulation. S22 will be kept with the knowledge of this deficiency.

\subsection{Comparison between 1, 2 and 11 injectors}

Averaged fields of temperature, heat release rate and fuel mass fraction are displayed at the centerplane of the injector in the transverse direction $(y=$ $-19.57 \mathrm{~mm}$ ) in Fig. 5, Fig. 6 and Fig. 7 for RCA1, RCA2 and RCA11, respectively. The cases RCA1 and RCA2 are showing very similar results where

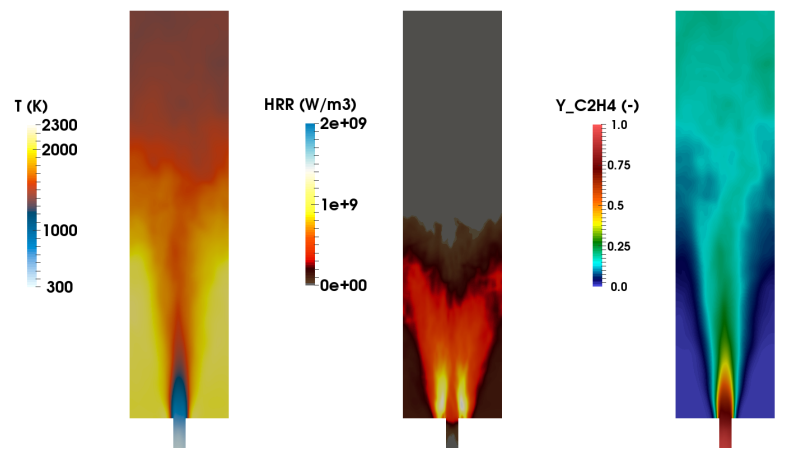

Fig. 5 Averaged temperature, heat release rate and fuel mass fraction at $y=-19.57 \mathrm{~mm}$ (injector center) for RCA1.

each injector is performing the same way. The injection jet is parallel to the direction of the injection and the resulted flame has a $\mathrm{V}$-shape with the same intensity. The case with eleven injectors RCA11 is performing differently, especially for the injection jets which are not parallel to each other. The combustion is also found more intense in the vicinity of the injectors located at the centerplane. The difference is most probably due to the side walls, which were prescribed as periodic in the cases RCA1 and RCA2, and which create here a transverse oscillatory mode which deflects the injection jets. In addition, the average temperature is also higher in RCA11 than in RCA1 or RCA2 by $100 \mathrm{~K}$ 


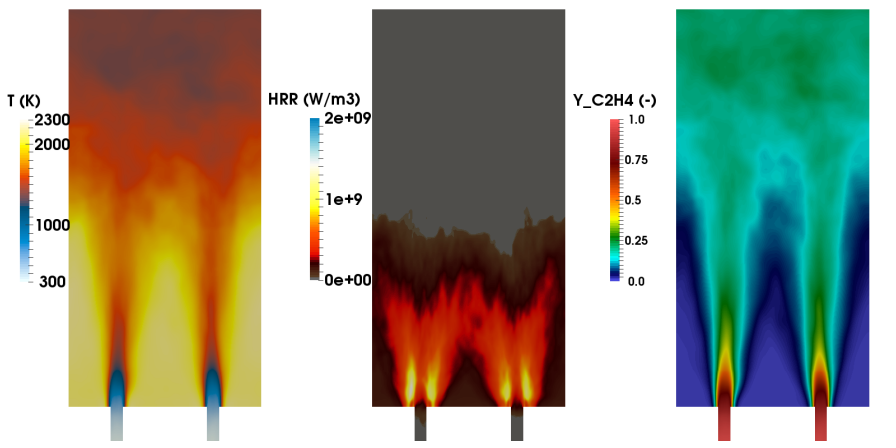

Fig. 6 Averaged temperature, heat release rate and fuel mass fraction at $y=-19.57 \mathrm{~mm}$ (injector center) for RCA2.

in the most reactive zones.

The simulations are then compared to the experimental data of Tuttle et al. [37] in the centerplane between two injectors (between 6-th and 7-th for RCA11). The averaged wall pressure is displayed in Fig. 8(a). RCA1 and RCA2 have very similar wall pressure, whereas the discrepancy between RCA1 and RCA11 is about $1 \mathrm{kPa}$. However, the experiment is fairly well predicted by all cases, except for the last point in the cavity ramp. The averaged streamwise and transverse velocities profiles are displayed in Fig. 9. The averaged streamwise velocity is still very well predicted by all the cases, only slight differences are observed between them. The simulations still over-predict the transverse velocity, regardless of the number of injectors involved. The difference between RCA1 and RCA2 is in average smaller than the one with RCA11, probably due to the periodic conditions prescribed at the side walls.

\subsection{Comparison between injectors of the case RCA11}

The eleven injectors case (RCA11) is showing some disparities on velocity, temperature and pressure profiles depending on the position of extraction. Comparisons have been done on the centerplanes between injectors 4-5, 6-7, 9-10, and 10-11 for averaged wall pressure inside the cavity (Fig. 8(b)), averaged temperature profiles and averaged velocity profiles (Fig. 10). In the region prior to the cavity, the wall pressure is higher at the centerplane between injectors 10 and 11, which is closer to the side wall. The wall pressure stays relatively the same for each centerplane in the cavity, except in the vicinity of the reattachment shock system where a higher wall pressure is found for the centerplane between injectors 4 and 5 and the lowest one between injectors 10 and 11. The discrepancy in temperature is slight between centerplanes 4-5, 9-10 and 10-11. But the centerplane near the central injector behaves differently. This centerplane has a higher temperature at the front of the cavity and a lower temperature at the rear of the cavity. The temperature discrepancy can reach $250 \mathrm{~K}$ in some regions. The combustion zone is in fact shifted from 

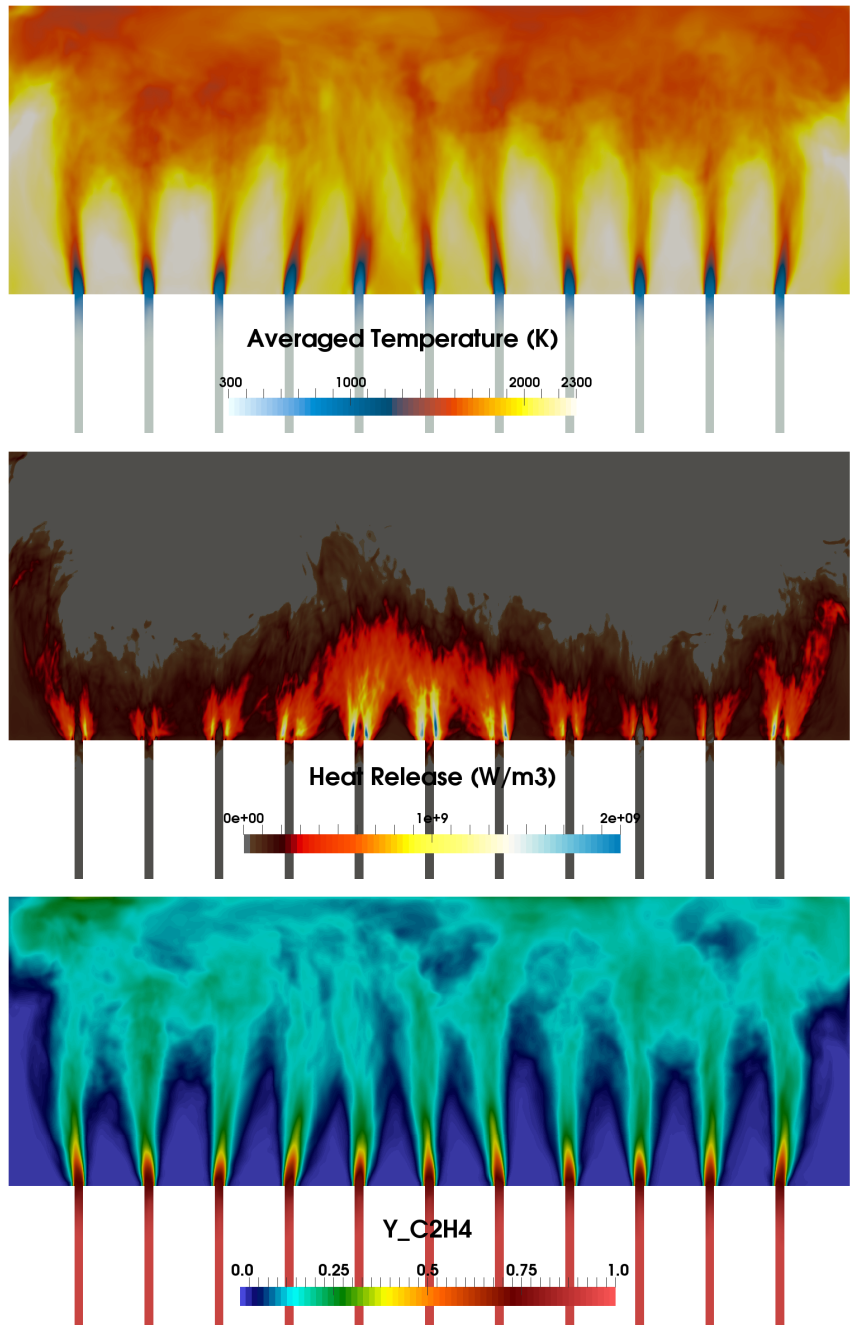

Fig. 7 Averaged temperature, heat release rate and fuel mass fraction at $y=-19.57 \mathrm{~mm}$ (injector center) for RCA11. Coarse mesh.

the large recirculation area to the small recirculation area in the centerplanes near the central injector (6-th), resulting in a difference in temperature. The averaged streamwise velocity is still not modified and stays relatively the same at each centerplane. The averaged transverse velocity is however dependent of the location of the centerplane, especially at the vicinity of the shock system.

The isosurface of temperature $(T=2000 \mathrm{~K})$ colored by the values of mixture fraction is displayed in Fig. 11. A lower temperature and a leaner mixture can be observed in the vicinity of the central injector. This indicates that the amount of cold airflow entering through the rear of the cavity is higher in 


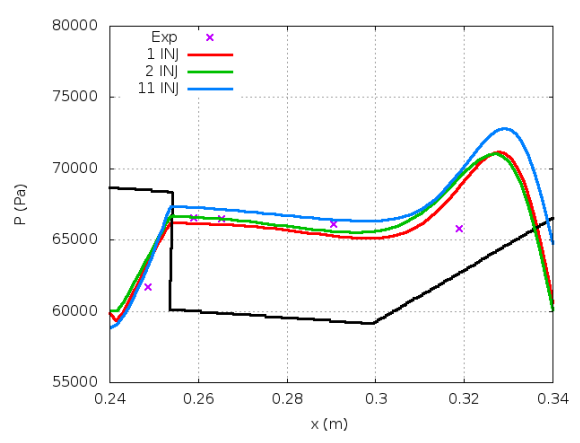

(a) Experiment (pattern), RCA1 (red line), (b) Experiment (pattern). RCA11: injectors RCA2 (green line) RCA11 (blue line, between 4-5 (red line), 6-7 (green line), 9-10 (blue line) 6-th and 7-th injector).

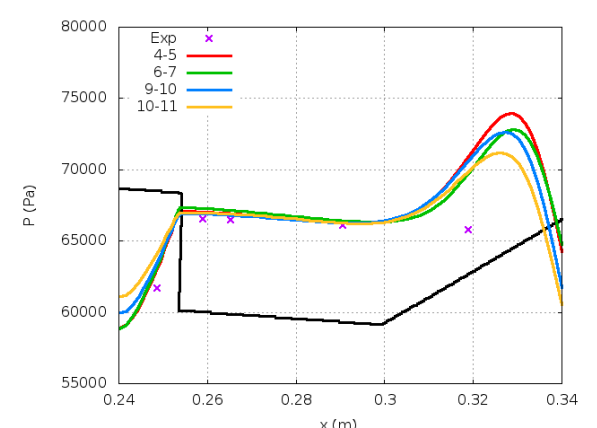
and 10-11 (yellow line).

Fig. 8 Averaged cavity wall pressure. Comparison between the numerical and the experimental data at the centerplane between two injectors.

the central injector region, thus decreasing the temperature and the mixture fraction. The origin of the airflow massively entering in the central injector region is difficult to capture and in the absence of more complete experimental results, it is too early to decide if this is a real feature of the experimental set-up or a numerical artefact. The differences between the simulation of the complete set-up and one or two injectors simulations can be considered unimportant if only stability issues or combustion regime analysis are considered. However to use simulation to fully characterize and design such a device, the full geometry must be included. Transporting a semi-detailed chemistry with its heavy load on memory and CPU time is then to be avoided. In the rest of the paper, the possibility of using a tabulated approach which allow to drastically decrease the CPU time $[8-10,20,27]$ to describe the chemistry is explored.

\section{A priori tabulated chemistry}

As discussed in Ruan et al. [34], the dominant combustion regime found in this device is the premixed regime. A tabulation of the chemistry based on a premixed flamelet library [29] without accounting for eventual compressibility effects has then be attempted and tested a priori on the results of the simulation with one injector presented in the previous section, but with a fine resolution (case RFA1) to minimize the impact of under resolution of the chemistry on the mesh.

\subsection{One dimensional laminar premixed flames}

One dimensional laminar premixed flame propagations were computed with the solver REGATH $[5,30,33]$ at $P=66 \mathrm{kPa}$ and $T=329 \mathrm{~K}$ in the flammability limits range of ethylene, which is $\phi \in[0.4,5]$, and over a distance of 

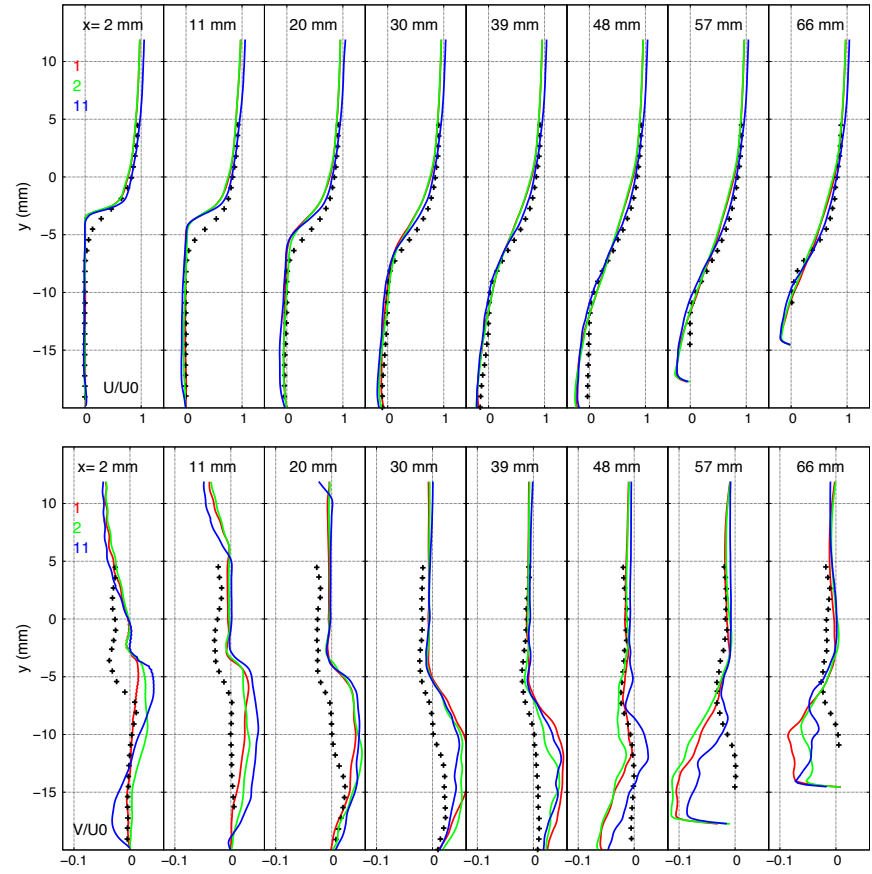

Fig. 9 Averaged streamwise (top) and tranverse (bottom) velocity profiles inside the cavity. Experiment (pattern), RCA1 (red line), RCA2 (green line) and RCA11 (blue line, between 6-th and 7-th) at the centerplane between two injectors.

10 meters. The purpose of this study is not to reach equilibrium with 1D flame propagation which in some cases require a computational domain of hundreds of kilometers, but to build a laminar premixed flame table which contains all the possible solutions of the LES. Therefore, the last point of each flamelet is in general not the equilibrium state.

The temperature profiles of laminar premixed flames at different equivalence ratios are displayed in Fig. 12. Superadiabatic temperature (SAT) phenomenon can be observed for high equivalence ratios and is identified by a temperature peak in the flame front which is higher than the adiabatic temperature. This phenomenon is present in hydrogen flames [12] and it has been investigated in hydrocarbon flames at rich mixtures numerically and experimentally by Liu et al. [19] for $\mathrm{C}_{1}-\mathrm{C}_{3}$ hydrocarbons and numerically by Meeks et al. [24] for $\mathrm{CH}_{4}$ and $\mathrm{C}_{2} \mathrm{H}_{2}$. Aside from the temperature peak, SAT is also associated with superequilibrium concentrations of some combustion products and with negative heat release rate (endothermic reactions). Meeks et al. [24] argue that SAT phenomenon is due to high gas velocities in the burning region at high equivalence ratios, while Babkin et al. [1] pointed out that SAT generally occurs due to competition between molecular diffusion and heat transfer processes. The right hand side figure of Fig. 12 is a close view of the temperature profiles for 

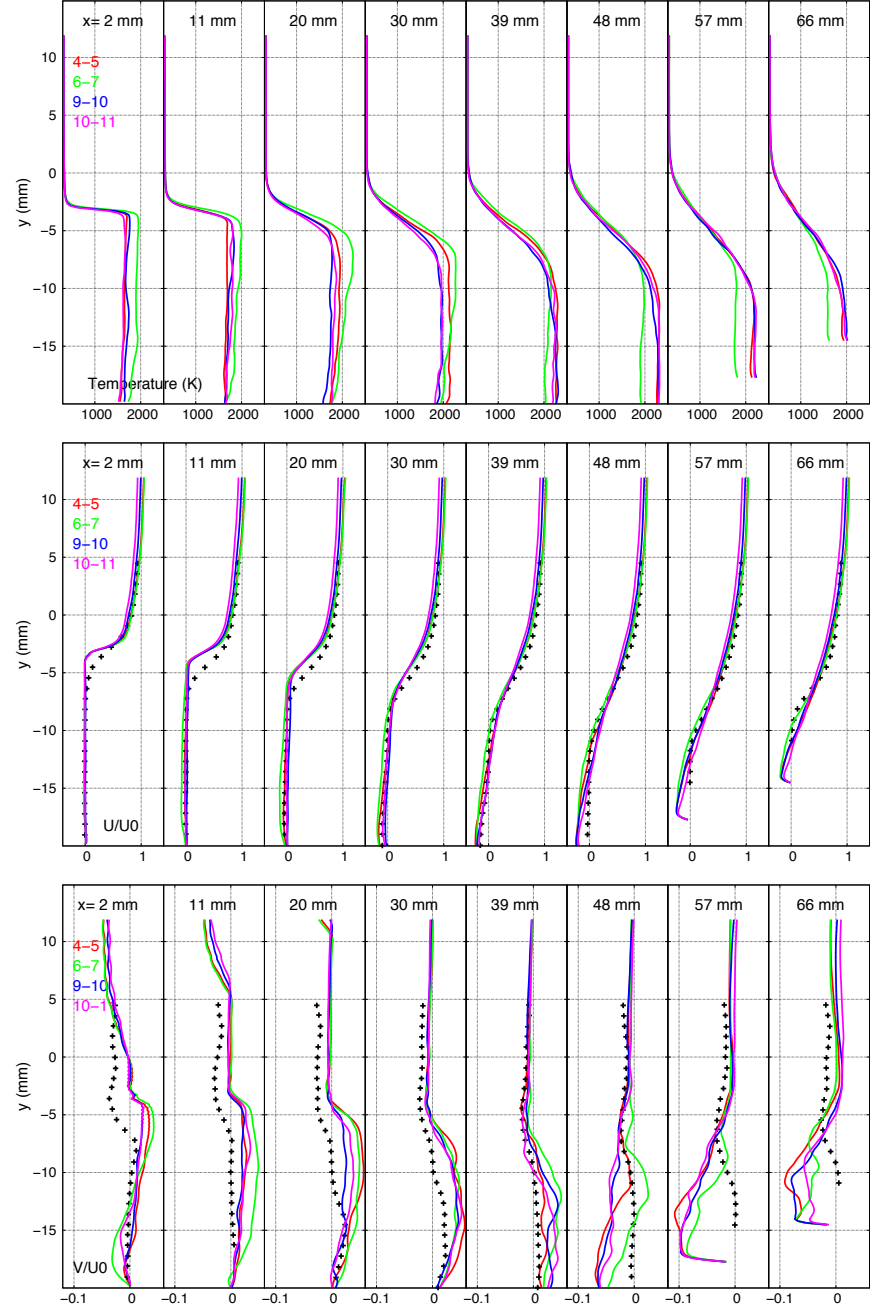

Fig. 10 Averaged temperature (top), streamwise (middle) and tranverse (bottom) velocity profiles inside the cavity, comparison between the numerical and the experimental data for coarse mesh at the centerplane between the two injectors for RCA11: experiment (pattern), injectors 4-5 (red line), 6-7 (green line), 9-10 (blue line) and 10-11 (magenta line).

equivalence ratio $\phi$ from 1.6 to 5 . In the case of ethylene-air combustion, SAT can be observed from $\phi=1.7$ with a slight temperature peak until $\phi=3.5$.

In order check at high equivalence ratios what would be the length needed in the 1D laminar premixed flamelet computed with REGATH to reach equilibrium as determined by EQUIL, the computational domain for the 1D laminar premixed flame has been extended to 5 kilometers for $\phi=4$. It is then verified that the equilibrium values for temperature and concentrations are reached (see Fig. 13). For higher equivalence ratios, an even longer domain would be 


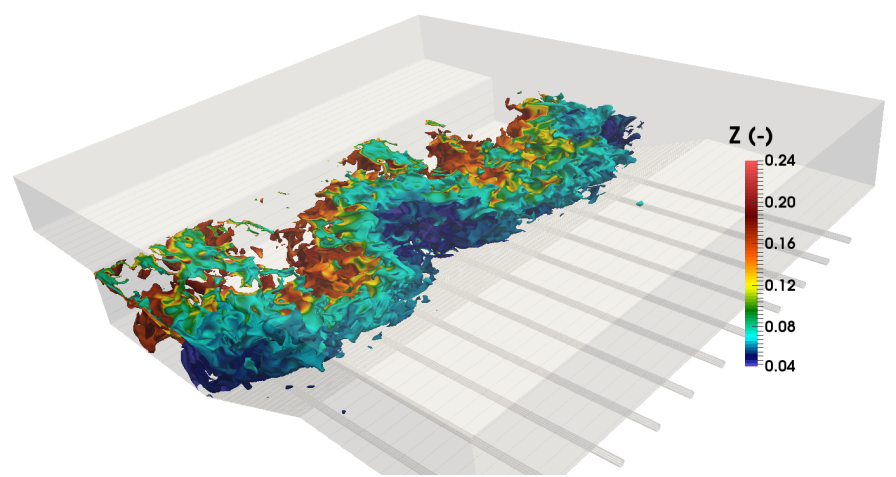

Fig. 11 Instantaneous isosurface of temperature at $2000 \mathrm{~K}$ for RCA11, colored by the values of mixture fraction.
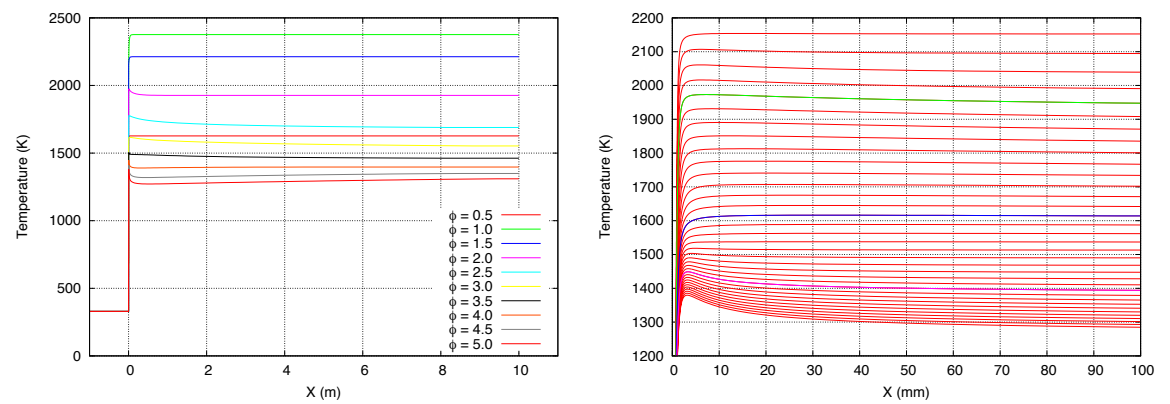

Fig. 12 Temperature profiles in a 1D laminar premixed flame for several equivalence ratios: $\phi \in[0.4,5]$ (left) and at rich compositions: $\phi \in[1.6,5]$ (right). On the right, the green line represents $\phi=2$, blue line for $\phi=3$ and purple line for $\phi=4$.

needed to reach equilibrium. As building a table with these very long domains would take for ever, a first table has been constructed with $\mathrm{v}$ for all equivalence ratios. However, it will be checked (section 3.3) that this is sufficient to contain all the compositions found in the LES, or if further refinements need to be done in some regions.

\subsection{Expressions of progress variable}

The definition of progress variable is a key point in tabulated chemistry and is not straightforward $[26,28,32]$. It is defined as the ratio of a linear combination of species mass fractions on the same quantity at equilibrium:

$$
C=\frac{Y_{C}}{Y_{C, e q}}
$$

So the progress variable $C$ takes value in $[0,1]$, with the extrema 0 and 1 corresponding to fresh gases and burnt gases at equilibrium, respectively. $Y_{C}$ can 

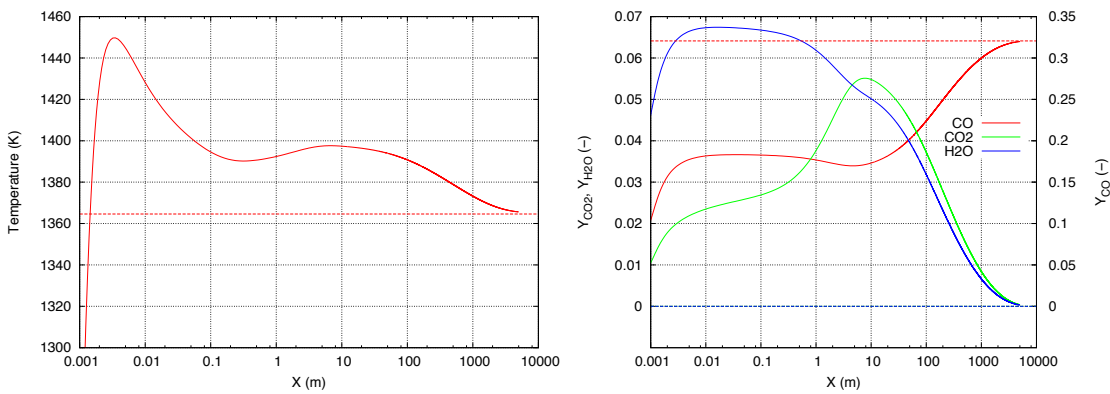

Fig. 13 Temperature (left) and main product mass fractions (right) along $\mathrm{X}$ for $\phi=4$. The dashed line represents the equilibrium value.

be defined either from the reactants plus $\mathrm{N}_{2}$ or from some major combustion products chosen carefully. In this work, we will be only interested in building the progress variable from the main products of ethylene-air combustion. Therefore, $Y_{C}$ will be constructed with $Y_{C O}, Y_{C_{2}}$ and $Y_{\mathrm{H}_{2} \mathrm{O}}$. Two expressions of $Y_{C}$ are studied and compared:

$$
\begin{aligned}
& Y_{C 1}=Y_{C O}+Y_{C_{2}} \\
& Y_{C 2}=Y_{C O}+Y_{C_{2}}+Y_{\mathrm{H}_{2} \mathrm{O}}
\end{aligned}
$$

The mass fractions at equilibrium could be taken from the 1D laminar premixed flame results if they are not far from the equilibrium, which is not the case in this study at high equivalence ratios. Thus, mass fractions from EQUIL [23] are used to compute $Y_{C, e q}$, in order to avoid discontinuities when prolongating the flame library outside the flammability limits. Verifications are shown in appendix A.

\subsection{Construction of laminar premixed tables}

Laminar premixed flame tables are generally constructed from 1D flames within the flammability limits and may be extended to all equivalence ratios by interpolation between fresh gases and equilibrium state. The advantage of using REGATH is that the solver can compute flames outside of the flammability limits, thus reducing the prolongation by interpolation which is not always appropriate. This method has already been investigated by Duboc et al. [8-10] for methane-air combustion by extending the table to $\phi=5.5$ (the flammability domain of methane-air is $\phi \in[0.6,1.4])$. The results were found to be more convincing than prolongation with equilibrium values. Here, the 1D flame simulations with REGATH have therefore been extended from $\phi_{\max }=5$ to 50 at $P=66 \mathrm{kPa}$ and $T=329 \mathrm{~K}$ with a computational domain of 10 meters long. The flame speed and the thermal flame thickness are displayed in Fig. 14. The highest speed and the lowest thickness are found for $\phi=1.17$ and worth respectively $s_{L, \max }=88 \mathrm{~cm} / \mathrm{s}$ and $\delta_{L, \min }=0.38 \mathrm{~mm}$ at 

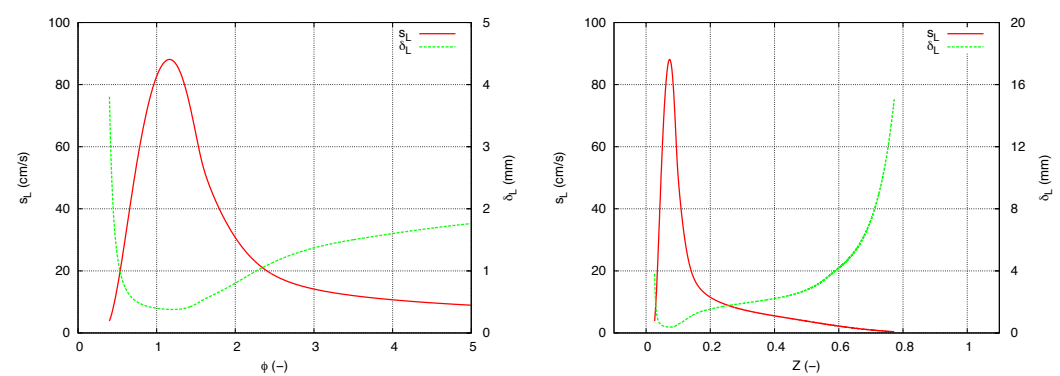

Fig. 14 Laminar premixed flame speed $\left(S_{L}\right)$ and thickness $\left(\delta_{L}\right)$ within the flammability limits (left) and in the whole computed range of equivalence ratios (right).

these conditions.

Look-up tables are created at $P=66 \mathrm{kPa}$ and $T=329 \mathrm{~K}$ for both expressions of progress variable with $\Delta C=0.002$ and for $\phi \in[0.4,50](Z \in[0.0265,0.773])$ with $\Delta \phi=0.01$. They are extended to the whole range of $Z$ by prolongation with equilibrium data of S22. Scatter plot of progress variables $\left(C_{1}\right.$ and $\left.C_{2}\right)$ versus mixture fraction $Z$ of LES results are shown in Fig. 15. Each point is
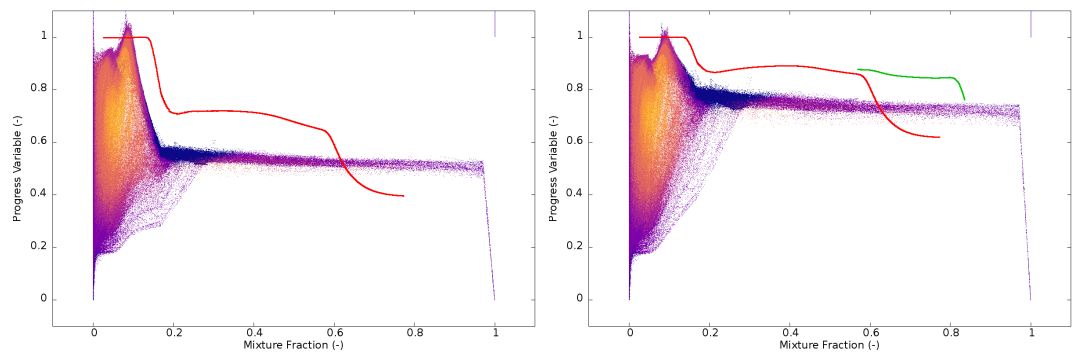

Fig. 15 Scatter plot of progress variables $C_{1}$ (a) and $C_{2}$ (b) vs. mixture fraction: blue color refers to negative heat release rate, the shading from purple to yellow represents cells with low to high heat release rate. The curves show the maximum value of tabulated progress variable for a given composition $C_{\max }(Z)$ with a $1 \mathrm{D}$ computational domain of $10 \mathrm{~m}$ for red color and $1 \mathrm{~km}$ for green color.

displayed with a color representing the intensity of heat release rate: blue for negative values and the shading from purple to yellow for low to high values. The region with negative heat release rate corresponds to the area with SAT (see Sec. 3.1), reactions are endothermic in this region in order to tend towards adiabatic temperature. The highest heat release rates are, as expected, found near the stoichiometric mixture $(Z=0.06366)$.

The red curves in Fig. 15 represent the last point $(X=10 \mathrm{~m})$ of each flamelet, as $C$ is an injective function of $X, C(X=10 \mathrm{~m})=\mathrm{C}_{\max }$, which means that all points below this curve are tabulated. There is a small region near $Z=0.1$ where the progress variable is superior to 1 . That phenomenon occurs more severely when the progress variable is not well defined, since one or several ma- 
jor species are missing to describe correctly this region, or when the number of points is not representative. The phenomenon of $C>1$ can be due to diffusion. The expression $C_{1}$ is therefore not valid in this study since the number of points with $C_{1}>1$ is significant compared to $C_{2}$ where less than $0.003 \%$ of cells are concerned. The table based on $C_{1}$ is consequently discarded, and $C_{2}$ is renamed as $C$ in the following.

Besides, the red curve starts to fall down for $Z>0.57(\phi>19.5)$ and goes under the LES results for $Z>0.6$, which means that the table does not contain those points. It needs to be extended to include higher progress variables. The prolongation can be done by interpolating between the last point of the flame and the equilibrium data, or by extending the computational domain in that region. The second technique is preferred in this work. The $1 \mathrm{D}$ computational domain has been extended to $1 \mathrm{~km}$ for $Z>0.57$ until $Z=0.835$, because a longer domain is required for higher mixture fraction to reach equilibrium. The new curve is represented in green color in Fig. 15.

At this stage, we would like to know whether the prolongation outside the flammability limits with non-propagating 1D flame (REGATH) gives more consistent results than the one with equilibrium prolongation. Three tables with different prolongations were built and compared to investigate that question. Their characteristics are shown in Tab. 2. As the temperature inside the

\begin{tabular}{clcc}
\hline \hline Table & 1D Flame for $\phi$ in & Prolongation for $\phi$ in & Temperature (K) \\
\hline TAB1 & {$[0.4,5]$} & {$[0,0.4[\cup] 5,+\infty[$} & 329 \\
TAB2 & {$[0.4,19.5]$} & {$[0,0.4[\cup] 19.5,+\infty[$} & 329 \\
TAB2b & {$[0.4,19.5]$} & {$[0,0.4[\cup] 19.5,+\infty[$} & 450 \\
TAB3 & {$[0.4,74.5]$} & {$[0,0.4[\cup] 74.5,+\infty[$} & 329 \\
\hline \hline
\end{tabular}

Table 2 Look-up table features. $P=66 \mathrm{kPa}$.

cavity is not constant and is always inferior to $450 \mathrm{~K}$ (for the non-reactive simulations), a fourth table (TAB2b) was constructed at $450 \mathrm{~K}$ and $66 \mathrm{kPa}$ to investigate the impact of the fresh gases temperature on the results. The flamelet libraries are displayed in Fig. 16.

\subsection{A priori comparison with LES field}

The a priori comparison is done on an instantaneous field for an adiabatic computation with one injector and a fine resolution (RFA1). The interpolation of the table data into the computational domain has been performed in the following way:

- First, the mixture fraction $Z$ and the progress variable $C$ are computed using the gas composition from the LES mass fractions $\left(Y_{k}\right)$;

- Then, using $Z$ and $C$ computed previously, the corresponding value of each tabulated quantity $\varphi_{t a b}$ is extracted. 

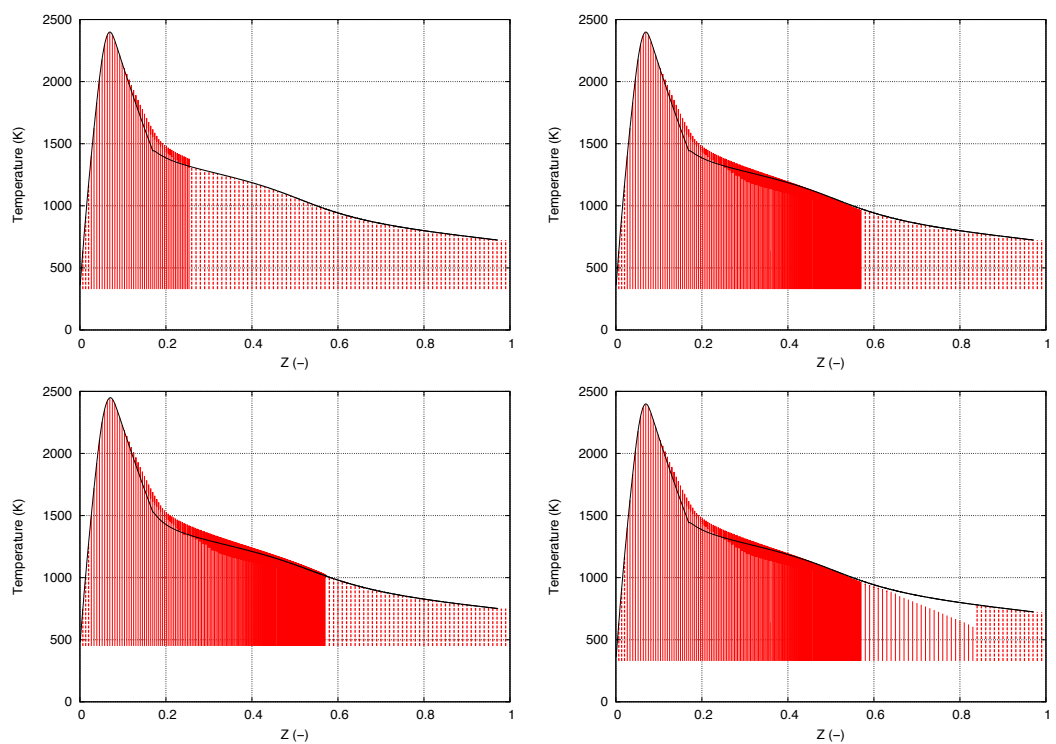

Fig. 16 Laminar premixed flamelet libraries: (a) TAB1, (b) TAB2, (c) TAB2b, (d) TAB3. Tabulated (-), interpolated (- - -).

In the few locations where $C>1$, possibly due to diffusion not accounted for in the table, the values are taken at $C=1$. The comparison has been done for the temperature and the main products mass fractions $\left(Y_{\mathrm{CO}}, Y_{\mathrm{CO}_{2}}, Y_{\mathrm{H}_{2} \mathrm{O}}\right)$. The instantaneous mixture fraction and progress variable for the case RFA1 are displayed in Figs. 17 and 18, respectively. The mixture is found very rich

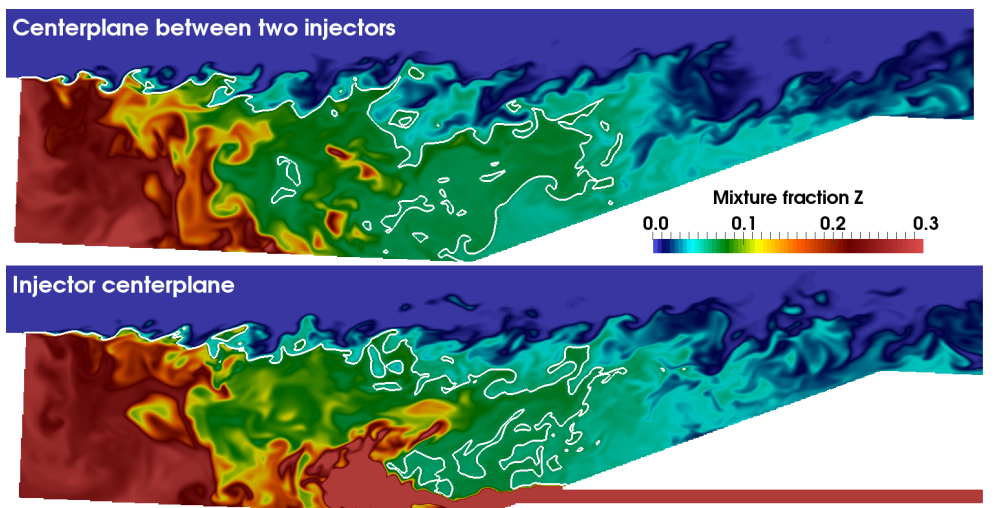

Fig. 17 Instantaneous field of mixture fraction inside the cavity: centerplane between two injectors (top) and injector centerplane (bottom). The stoichiometric line is displayed in white color.

at the front of the cavity $(Z>0.2)$, and lean to moderately rich in the large 


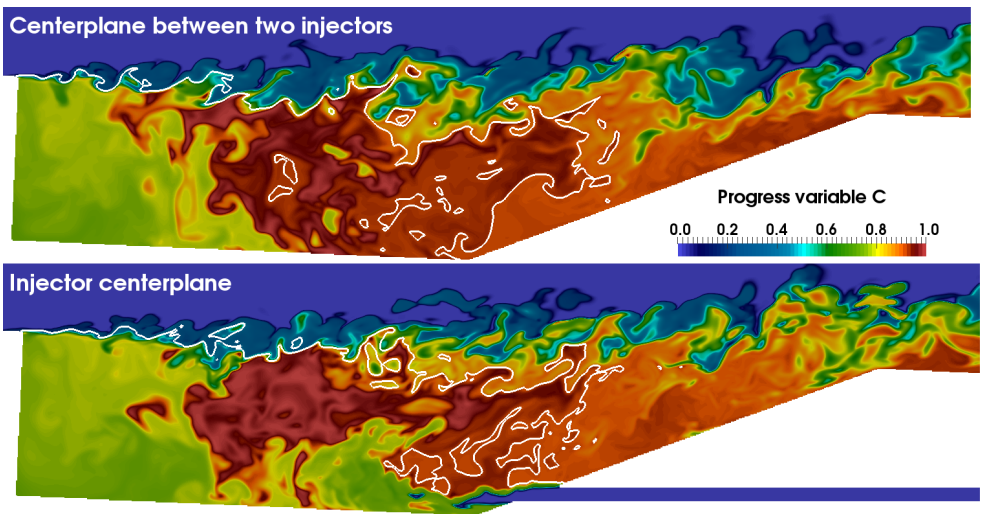

Fig. 18 Instantaneous field of progress variable inside the cavity: centerplane between two injectors (top) and injector centerplane (bottom). The stoichiometric line is displayed in white color.

recirculation area $(Z \in[0.03,0.1])$, except at the exit of the injector where the mixture fraction is expected to have very high values. On the other hand, the progress variable is globally high in the whole cavity with $C>0.7$. Values of $C$ higher than 0.9 are found in the large recirculation area, while the small recirculation area is having much smaller values of progress variable $C \approx 0.75$. This result is actually expected, since the time required to reach equilibrium increases strongly with large values of mixture fraction.

The instantaneous temperature field is displayed in Fig. 19 for cuts in the spanwise direction $(z)$ and in Fig. 20 for cuts in the streamwise $(x)$ and the transverse $(y)$ directions for LES results and laminar premixed table (TAB2) interpolations. The $z$-cuts are still taken at the injector centerplane and the centerplane between two injectors. The first $y$-cut is localized in the mixing layer, while the second corresponds to the middle line of the cavity. The $x$-cuts are from the interface between the recirculation zones $(x=20 \mathrm{~mm}$ from the cavity front) and the middle line of the large recirculation area $(x=39 \mathrm{~mm})$. At the first glance, temperatures from LES and TAB2 appear very close to each other. The only noticeable difference lies in the small recirculation area where TAB2 underpredicts the temperature. In order to better quantify the discrepancies detected in the instantaneous temperature fields, values at eight positions inside the cavity with constant $x$ are picked up. These positions are the same as those used for the velocity profiles which are: $x=2,11,20,30,39$, $48,57,66 \mathrm{~mm}$ from the cavity front. The mixture fraction and the progress variable profiles are first displayed in Fig. 21. The mixing appears efficient and the mixture fraction presents smooth variations. The progress variable is more affected by the turbulence and then features strong spatial gradients. The temperature and the $\mathrm{H}_{2} \mathrm{O}$ mass fraction profiles for TAB1, TAB2 and TAB3 are displayed in Fig. 22 and compared to the LES values in the injector centerplane. Readers are reminded that the only difference between these three tables is the way the prolongation outside the flammability limits was 

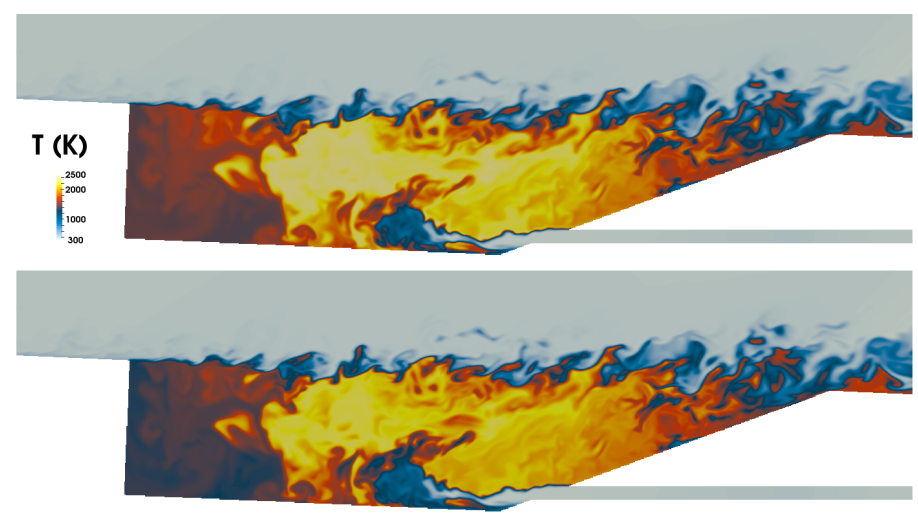

(a) Injector centerplane

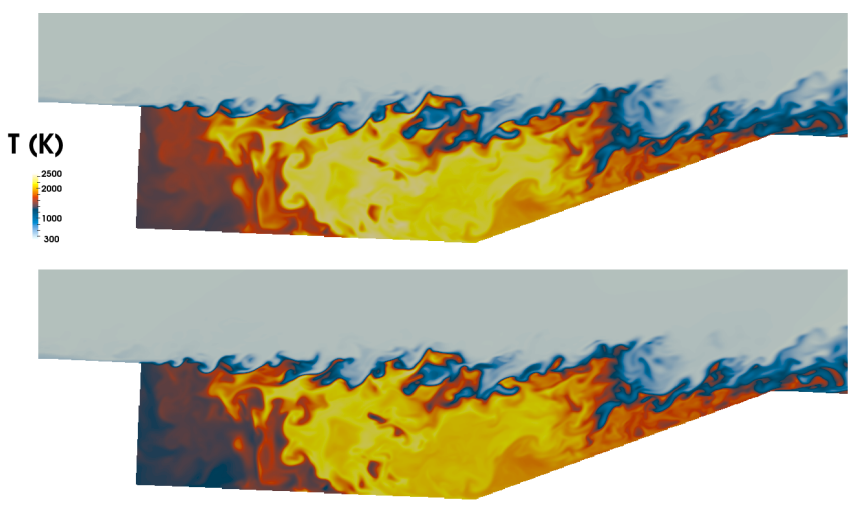

(b) Centerplane between two injectors

Fig. 19 Temperature comparison with LES results: LES (top), TAB2 (bottom).

done (see Tab. 2 of the previous section). TAB1 has some shortcomings in predicting the temperature or the $\mathrm{H}_{2} \mathrm{O}$ mass fraction outside the flammability limits. The two other tables do not have this issue and have very similar results. The only difference between TAB2 and TAB3 lies in a small region at the exit of the injector where TAB3 has more accurate results. This analysis shows that table prolongation with equilibrium hypothesis is not accurate in the current simulation and it is preferable to use 1D laminar premixed flames even outside the flammability limits. Compared to the LES, the temperature from the table is weaker at the front of the cavity by about $100 \mathrm{~K}$, and in regions with strong variations of mixture fraction where the discrepancy can reach $200 \mathrm{~K}$. The differences in $\mathrm{H}_{2} \mathrm{O}$ mass fraction can be related to the difference in temperature.

A comparison between TAB2 and TAB2b has been performed to investigate the impact of initial temperature of the laminar premixed flame tables. Again, 

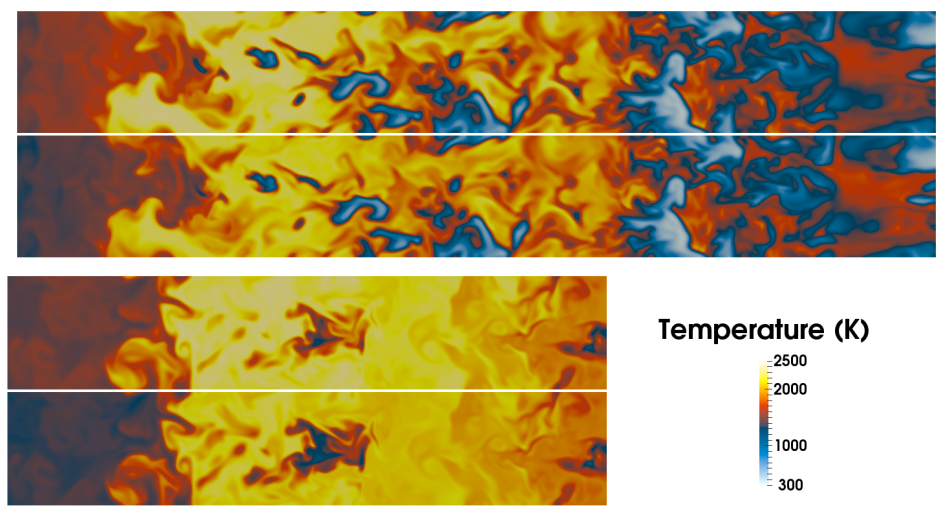

Temperature (K)

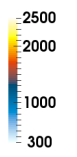

(a)
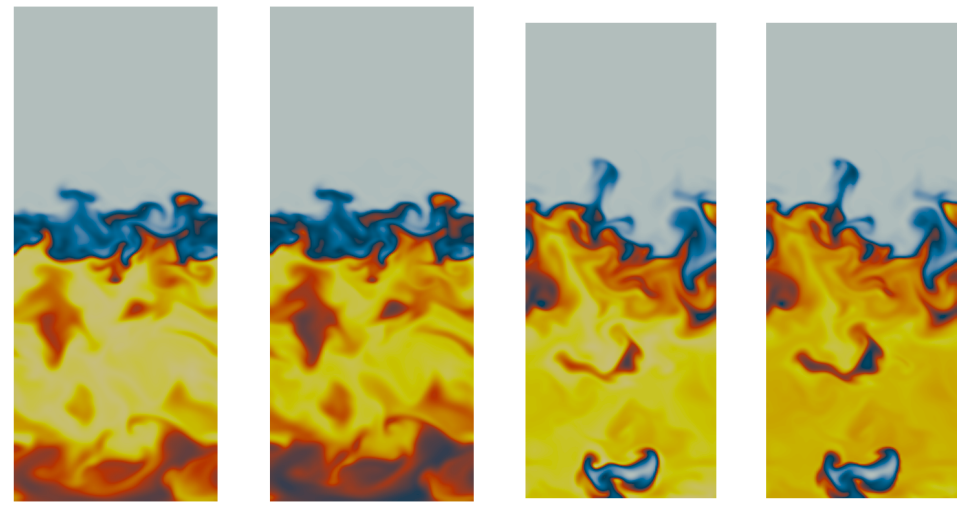

(b)

Fig. 20 Temperature comparison with (a) constant y cuts at mixing layer $(y=-7 \mathrm{~mm})$ and middle line of the cavity $(y=-14 \mathrm{~mm}$ ): from top to bottom, LES, TAB2 at $y=-7 \mathrm{~mm}$, then LES, TAB2 at $y=-14 \mathrm{~mm}$; and (b) constant $\mathrm{x}$ cuts at the interface of the recirculation zones $(x=20 \mathrm{~mm}$ from the cavity front) and the middle of the large recirculation area $(x=39 \mathrm{~mm})$ : from left to right LES, TAB2 at $x=20 \mathrm{~mm}$, then LES, TAB2 at $x=39 \mathrm{~mm}$.

temperature and $\mathrm{H}_{2} \mathrm{O}$ mass fraction are displayed in Fig. 23 for the injector centerplane. The temperature of the freestream in the case TAB2b is higher because the temperature of the fresh gases is $450 \mathrm{~K}$ instead $329 \mathrm{~K}$. TAB2b is having slightly better performance than TAB2 inside the cavity, but the opposite occurs when it comes to the mixing layer. Actually, the average temperature field of the non-reactive case has shown that only the inner cavity is featuring temperatures around $450 \mathrm{~K}$, the temperature in the mixing layer is lower and approaching the freestream temperature (329 K). An appropriate way would be building a table including compressibility effects. Besides, there is almost no difference for the mass fraction of $\mathrm{H}_{2} \mathrm{O}$ between TAB2 and TAB2b. The initial temperature of the laminar premixed flames has only a slight im- 

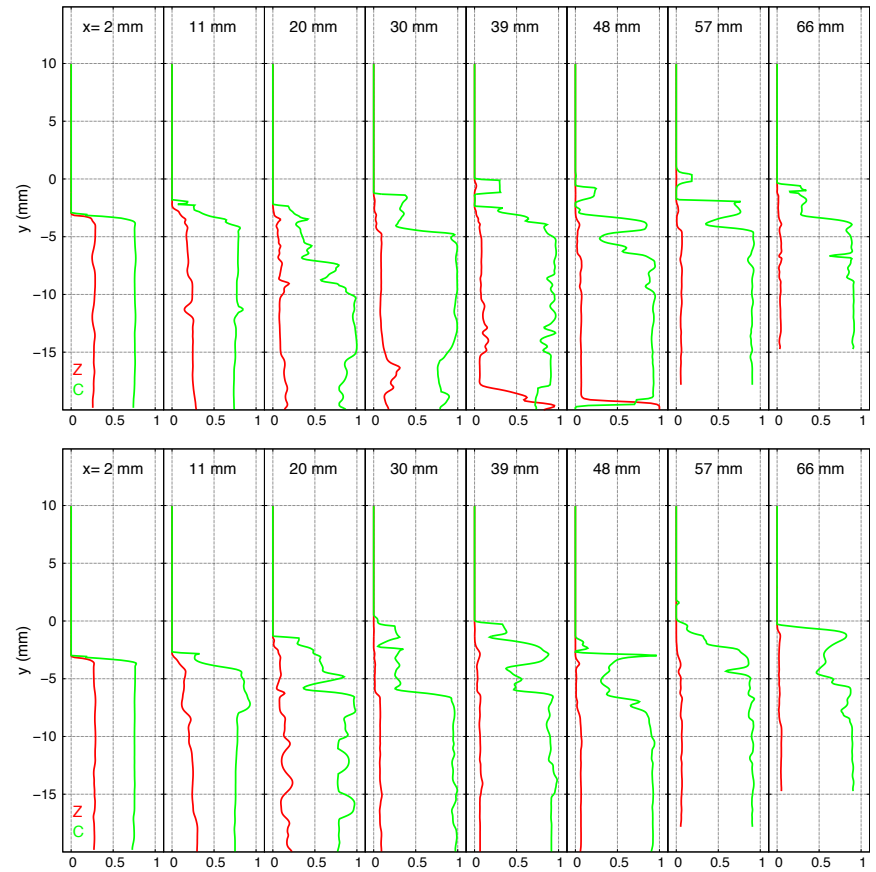

Fig. 21 Instantaneous mixture fraction and progress variable profiles inside the cavity in (top) the injector centerplane, and (bottom) the centerplane between two injectors.

pact on the results suggesting that compressibility effect can be neglected in that device. TAB3 will be used for the next comparisons.

Profiles of mass fractions of $\mathrm{CO}$ and $\mathrm{CO}_{2}$ for LES and TAB3 are displayed in Figs. 24 and 25 respectively in the injector centerplane and in the centerplane between two injectors. The slight discrepancies that were observed for the temperature in the injector centerplane are increased for the mass fractions of $\mathrm{CO}$ and $\mathrm{CO}_{2}$. The difference stays relatively low at the large recirculation area for the profiles in the centerplane between two injectors, except at the rear of the cavity $(x=66 \mathrm{~mm})$. At the front of the cavity, TAB3 and the LES are totally different regardless of the centerplane. Actually, when $Y_{C O}$ is overpredicted, $Y_{\mathrm{CO}_{2}}$ is underpredicted at the same area. The sum of both mass fractions $Y_{\mathrm{CO}}+Y_{\mathrm{CO}_{2}}$ is displayed in Fig. 26. The fitting is nearly perfect in the large recirculation area, but still some discrepancies persist in the small recirculation area $(x=2,11 \mathrm{~mm})$ and in the interface between the recirculation zones $(x=20 \mathrm{~mm})$. The reason for the differences found between LES and TAB3 can be due to several factors that are be discussed in the next section. 

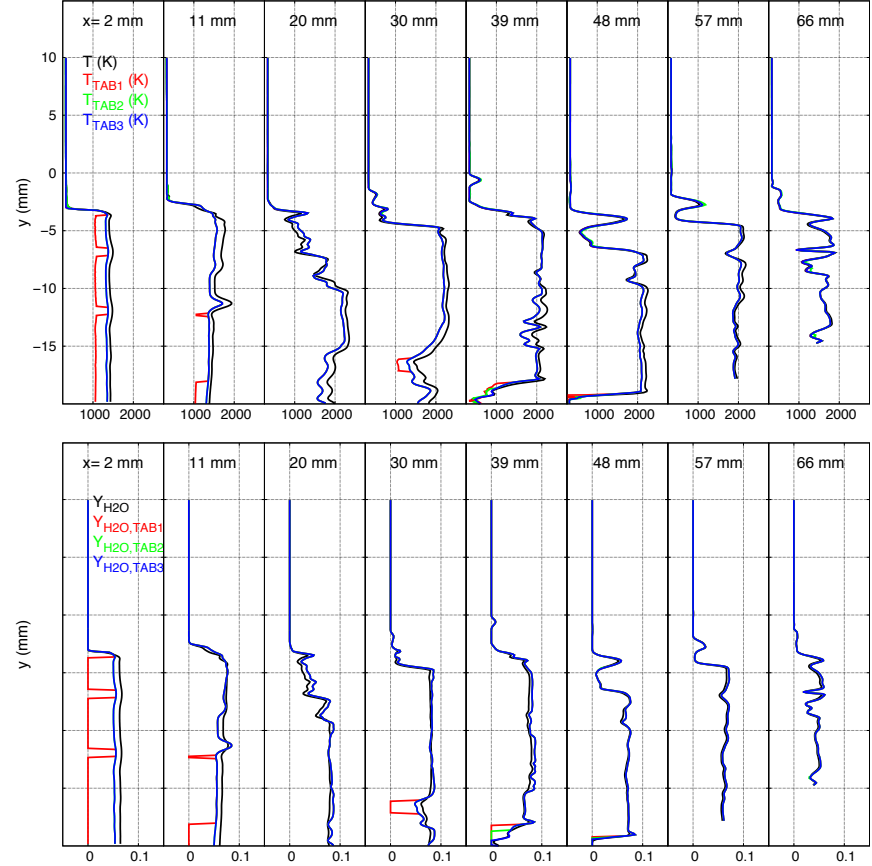

Fig. 22 Instantaneous (top) temperature and (bottom) mass fraction of $\mathrm{H}_{2} \mathrm{O}$ profiles inside the cavity in the injector centerplane: comparison between LES and tables with different prolongation methods outside the flammability limits.

\subsection{Origin of discrepancies between tabulated approach and LES}

Dilution by burnt gases, nonpremixed flame regime or strong scalar dissipation are the most probable origin of the discrepancies observed between tabulation and transported chemistry. These three options are investigated in the following.

\subsubsection{Dilution by burnt gases}

The dilution rate is the ratio of the burnt gases in the mixture and is defined as:

$$
\mathcal{D}_{r}=\frac{Y_{B G}}{1-Y_{N_{2}}}
$$

where $Y_{B G}$ is the mass fraction of the burnt gases. A species is tagged as burnt gas when it is neither a hydrocarbon $\left(\mathrm{C}_{n} \mathrm{H}_{m}\right)$ or a species from air $\left(\mathrm{O}_{2}\right.$ or $\left.\mathrm{N}_{2}\right)$ :

$$
Y_{B G}=1-Y_{C_{n} H_{m}}-Y_{O_{2}}-Y_{N_{2}}
$$

The instantaneous field of dilution rate is displayed in Fig. 27 in the injector centerplane and in the centerplane between two injectors. The whole cavity 

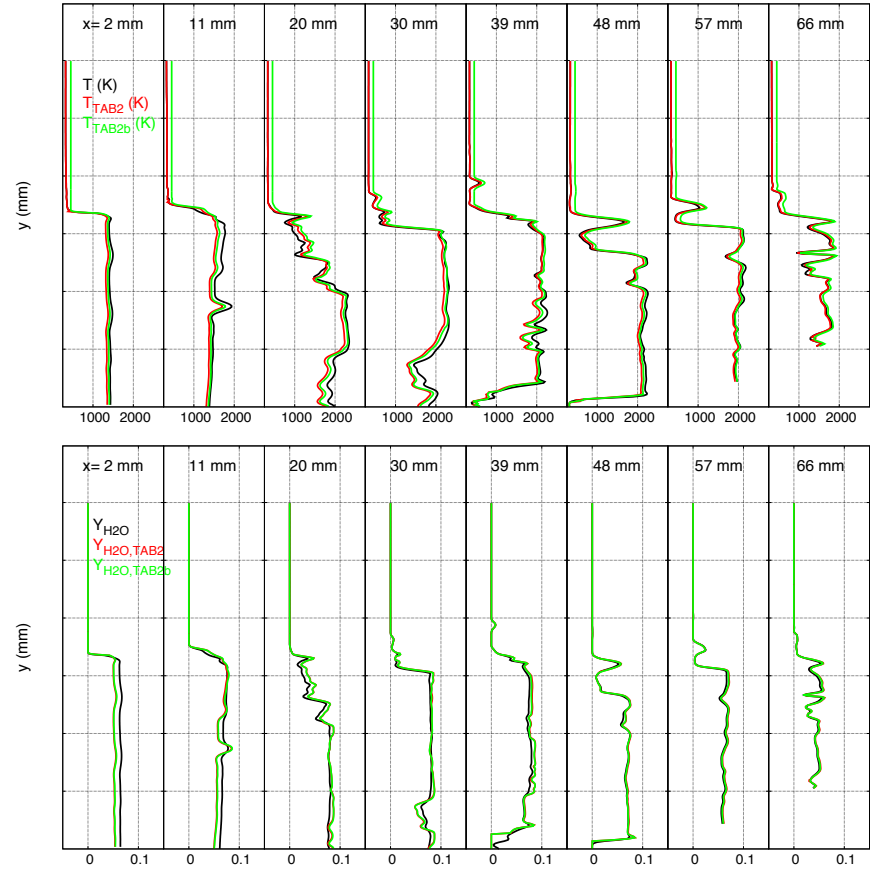

Fig. 23 Instantaneous (top) temperature and (bottom) mass fraction of $\mathrm{H}_{2} \mathrm{O}$ profiles inside the cavity in the injector centerplane: comparison between LES and tables with different initial temperatures.

is strongly diluted by burnt gases with $\mathcal{D}_{r}>0.5$. The front of the cavity is roughly composed of half burnt gases and half fuel $\left(\mathcal{D}_{r} \in[0.5,0.7]\right)$. The dilution rate is the highest in the large recirculation area where only burnt gases are present $\left(\mathcal{D}_{r}>0.9\right)$. Finally, the cavity ramp is featuring dilution rate from 0.4 to 0.7 because of the air entering at this area. Strong dilution is found everywhere inside the cavity, and yet the comparison between LES and TAB3 was satisfying in the most parts of the cavity, therefore, dilution could only be the origin of some minor discrepancies between the LES and the tabulated chemistry. Dilution without heat losses has in fact no impact on premixed flamelet properties [38].

\subsubsection{Nonpremixed flame regime}

The instantaneous heat release rate and the flame index are displayed in Fig. 28 at four cuts in the streamwise direction. The cuts are representing the area of the small recirculation zone $(x=2 \mathrm{~mm})$, the interface between the recirculation zones $(x=20 \mathrm{~mm})$, the large recirculation zone $(x=39 \mathrm{~mm})$ and the rear of the cavity $(x=66 \mathrm{~mm})$. No combustion is found in the small recirculation zone, so the burnt gases of this region come from the interface between the recirculation zones where the combustion regime is mostly non- 

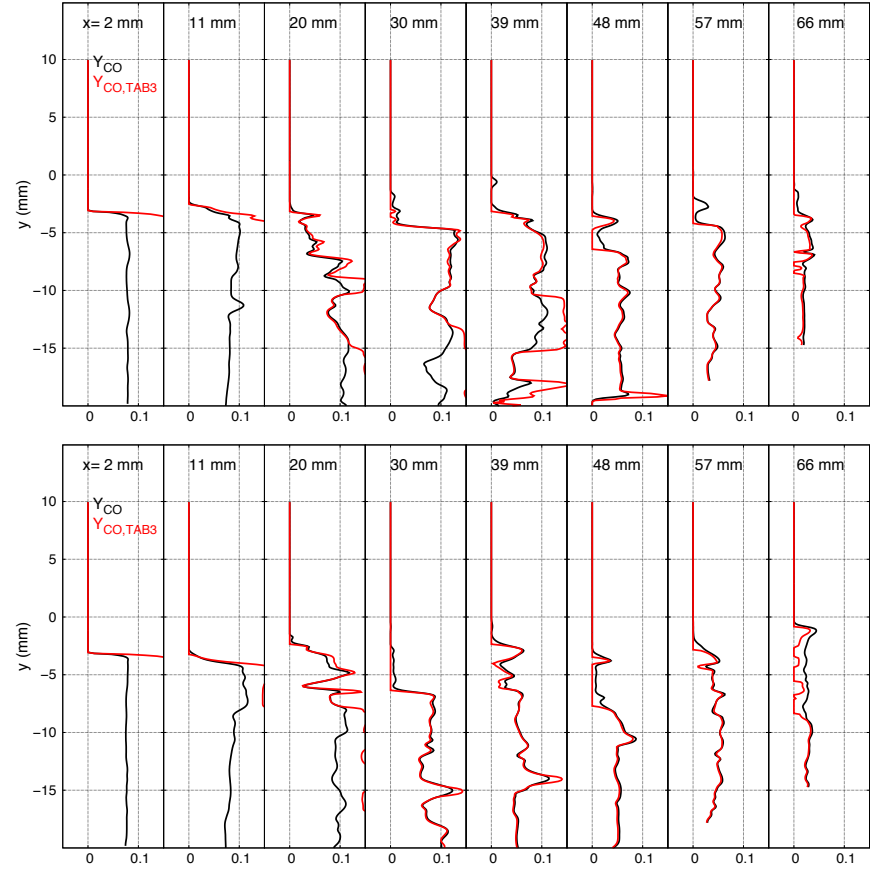

Fig. 24 Instantaneous CO mass fraction profiles inside the cavity in the injector centerplane (top) and the centerplane between two injectors (bottom): comparison between LES and TAB3.

premixed. Trying to predict a nonpremixed flame with a premixed flame table is expected to be improper. However, nonpremixed combustion is also found in the large recirculation area, and the premixed table is accurate in that region. Fiorina et al. [11] found that a laminar premixed flame tabulation (FPI solver) could be efficient in predicting nonpremixed flames if the mixture fraction is close to the stoichiometric value. Indeed, chemistry near stoichiometry is too fast for any diffusive processes to have an impact on it, and thus becomes independent of the flame configuration. The mixture fraction in the large recirculation area is between 0.05 and 0.10 which is close to the stoichiometric value $\left(Z_{s t}=0.06366\right)$, chemistry is well predicted by the premixed flame tabulation regardless of the combustion regime. On the other hand, the interface between the recirculation zones is featuring mixture fractions of 0.15 to 0.25 , chemistry is slow in that region so diffusive effects can intervene and make the species concentration dependent of the flame configuration.

Another reason would be that the small recirculation area is acting like a perfectly stirred reactor (PSR), and the time required to reach the values given by the premixed flame tabulation is too long to be achieved, since the residence time of this region is only a few milliseconds [34]. The mixture fraction in the small recirculation area being almost constant, a mixture composition at $Z=0.279$ is then extracted from the LES and used as an initial condition 

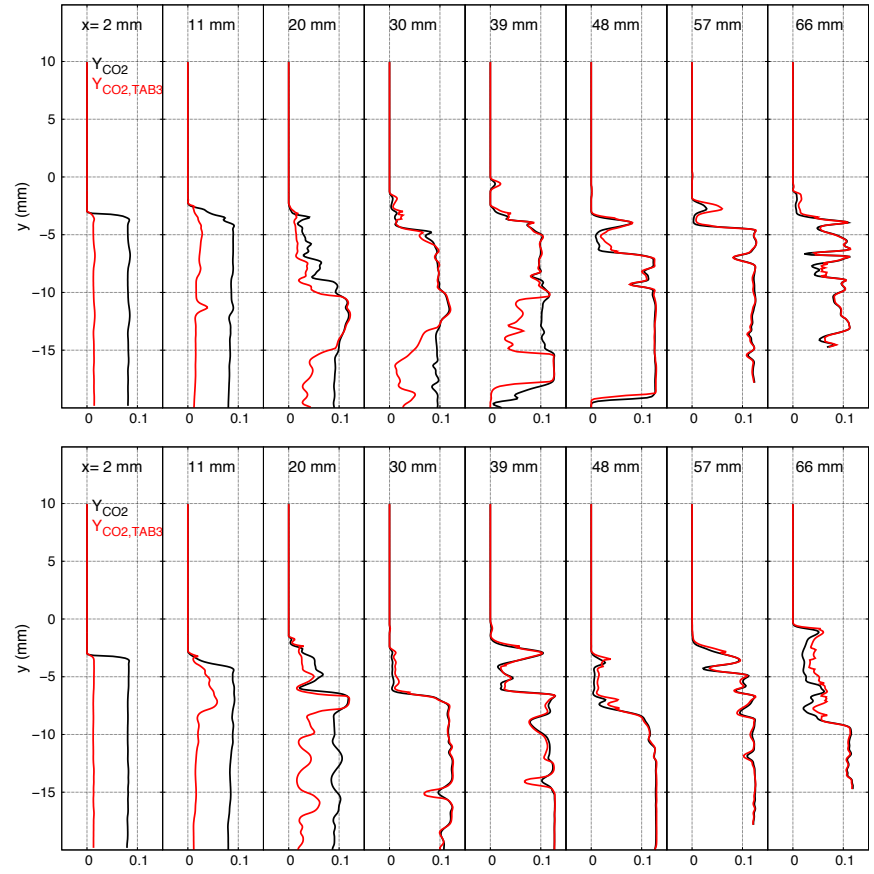

Fig. 25 Instantaneous $\mathrm{CO}_{2}$ mass fraction profiles inside the cavity in the injector centerplane (top) and the centerplane between two injectors (bottom): comparison between LES and TAB3.

for PSR computations with SENKIN [22]. The mass fractions of $\mathrm{CO}$ and $\mathrm{CO}_{2}$ are 0.078 and 0.081 respectively for the LES, and 0.158 and 0.013 respectively for TAB3. The mass fractions of $\mathrm{CO}$ and $\mathrm{CO}_{2}$ after $100 \mathrm{~s}$ physical time computation are displayed in Fig. 29. The mass fraction of $\mathrm{CO}$ is increasing while the one of $\mathrm{CO}_{2}$ is decreasing, then evolving towards the values of TAB3. However, even $100 \mathrm{~s}$ is not sufficient for the mass fractions to reach $Y_{C O, T A B 3}$ and $Y_{\mathrm{CO}_{2}, T A B 3}$. A premixed flame tabulation could never predict the burnt gas composition in this region which is fed by the rich side of a diffusion burning flame structure.

The discrepancies at the front of the cavity being clarified, we are now interested in those in the large recirculation zone, which are probably due to strong scalar dissipations.

\subsubsection{Scalar dissipations}

Three scalar dissipations are now investigated in this section. They are the mixture fraction dissipation $\chi_{Z}$, the progress variable dissipation $\chi_{Y_{C}}$ and the 

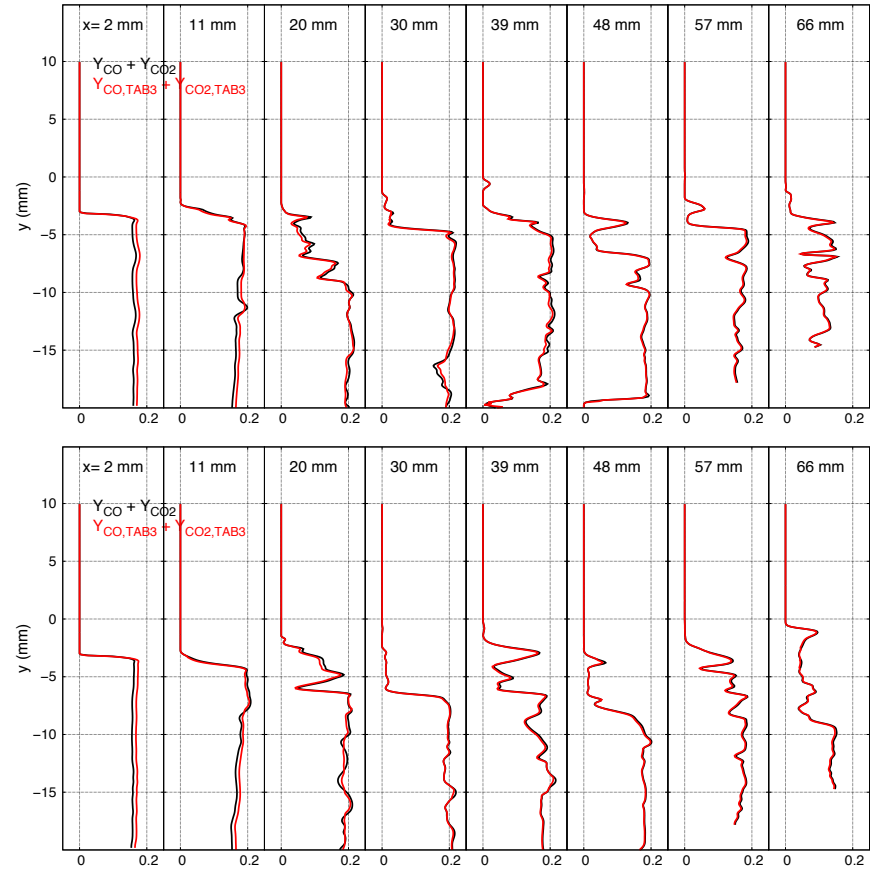

Fig. 26 Instantaneous $Y_{\mathrm{CO}}+Y_{\mathrm{CO}_{2}}$ profiles inside the cavity in the injector centerplane (top) and the centerplane between two injectors (bottom): comparison between LES and TAB3.
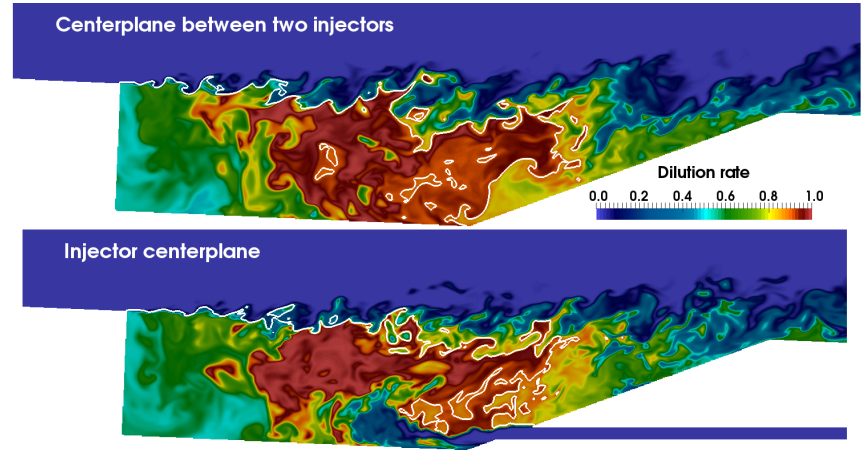

Fig. 27 Instantaneous field of dilution rate $\left(\mathcal{D}_{r}\right)$ inside the cavity for RFA1: centerplane between two injectors (top) and injector centerplane (bottom). The stoichiometric line is displayed in white color.

cross scalar dissipation $\chi_{Z C}$ :

$$
\left\{\begin{aligned}
\chi_{Z} & =D_{Z}|\nabla Z|^{2} \\
\chi_{Y_{C}} & =D_{Y_{C}}\left|\nabla Y_{C}\right|^{2} \\
\chi_{Z C} & =D_{Z C}|\nabla Z|\left|\nabla Y_{C}\right|
\end{aligned}\right.
$$



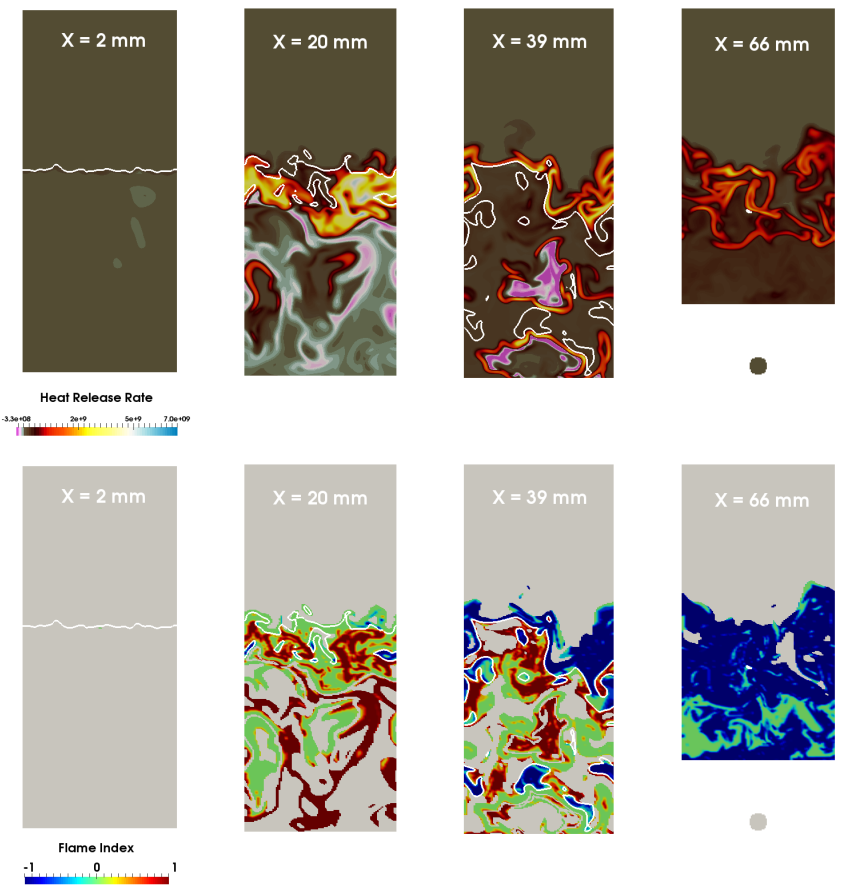

Fig. 28 Instantaneous heat release rate (top) and flame index (bottom) in the small recirculation zone $(x=2 \mathrm{~mm})$, the interface between the recirculation zones $(x=20 \mathrm{~mm})$, the large recirculation zone $(x=39 \mathrm{~mm})$ and the rear of the cavity $(x=66 \mathrm{~mm})$. The stoichiometric line is represented in white color. Case RFA1.

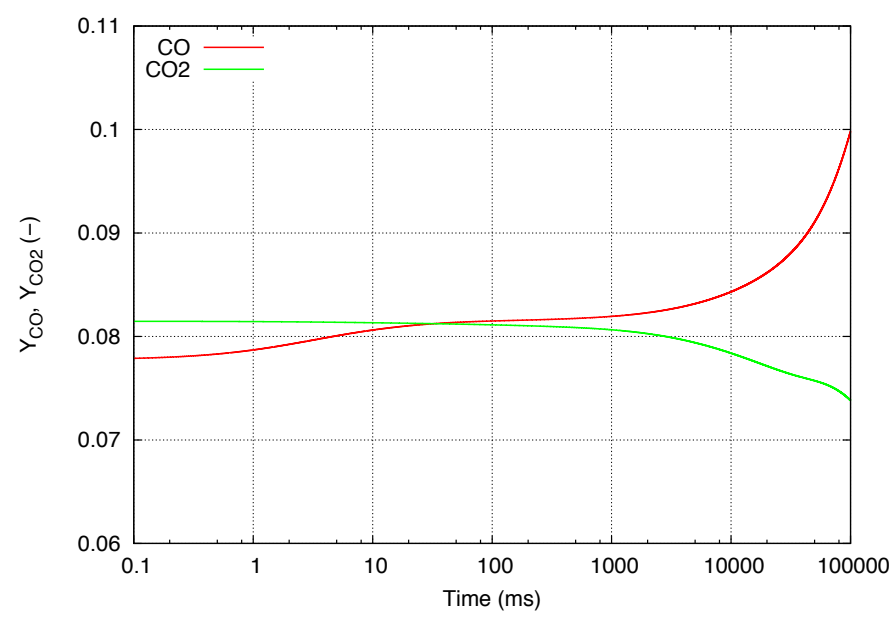

Fig. 29 Evolution in time of $\mathrm{CO}$ and $\mathrm{CO}_{2}$ mass fractions, computed with SENKIN [22]. 
where the coefficients $D_{Z}, D_{Y_{C}}$ and $D_{Z C}$ are:

$$
\left\{\begin{aligned}
D_{Z} & =\frac{\lambda}{\rho C_{p}} \\
D_{Y_{C}} & =\frac{D_{C O}\left|\nabla Y_{C O}\right|+D_{C O_{2}}\left|\nabla Y_{C O_{2}}\right|+D_{H_{2} O}\left|\nabla Y_{H_{2} O}\right|}{\left|\nabla Y_{C}\right|} \\
D_{Z C} & =\sqrt{D_{Z} D_{Y_{C}}}
\end{aligned}\right.
$$

Instantaneous fields of these scalar dissipations are displayed in Fig. 30 for cuts in the injector centerplane and the centerplane between two injectors, in Fig. 31 for cuts in the streamwise direction showing planes at the small recirculation zone $(x=2 \mathrm{~mm})$, the interface between the recirculation zones $(x=20 \mathrm{~mm})$, the large recirculation zone $(x=39 \mathrm{~mm})$ and the rear of the cavity $(x=66 \mathrm{~mm})$. Strong dissipation of the mixture fraction is found at the exit of the injector, following the path of the fuel injection. The mixing layer above the small recirculation zone and the interface between the recirculation zones are featuring scalar dissipations of moderate intensity. The difference between $\chi_{Z}$ and $\chi_{Y_{C}}$ is that the dissipation of the progress variable is weak at the interface between the recirculation zones and moderately strong in the whole mixing layer. The scalar dissipations profiles in Fig. 32 show that the large discrepancies between LES and TAB3 for $Y_{\mathrm{CO}}$ and $Y_{\mathrm{CO}_{2}}$ (see Fig. 24 and 25) in the large recirculation zone occur in zones with strong dissipation of mixture fraction. The dissipation of progress variable in the mixing layer only leads to small differences between LES and TAB3.

In order to link the scalar dissipation to the combustion regime, the regions with high values of $\chi_{Z}$ in the injector centerplane at the fourth and fifth cuts $(x=30$ and $39 \mathrm{~mm}$, respectively) have been investigated along with the flame index (Fig. 33). In the fourth cut, strong scalar dissipations are located in regions with rich premixed combustion regime $(F I=1)$. While in the fifth cut, the strongest dissipations are found at the exit of the injector $(y \in[-20,-17] \mathrm{mm})$ with nonpremixed combustion regime $(F I=0)$. At the middle of cavity $(y>-15 \mathrm{~mm})$, rich premixed and nonpremixed combustion regimes are found in zones with moderately strong scalar dissipations. The values of $\chi_{Z}$ are insignificant for areas with lean premixed combustion regime $(F I=-1)$ in both cuts. The largest discrepancies between the LES and TAB3 are actually located in regions with moderate scalar dissipations and featuring premixed combustion regime (see temperature profiles in Fig. 22). Regions in the large recirculation area with nonpremixed combustion are well predicted for the temperature, even with strong scalar dissipations.

In rich premixed regions with moderate scalar dissipations, solely the concentrations of $\mathrm{CO}$ and $\mathrm{CO}_{2}$ are largely affected, the mass fractions of $\mathrm{H}_{2} \mathrm{O}$ between LES and TAB3 were found to be very close to each other. A study on the sensitivity of these species to the gradient of equivalence ratio has been performed for one dimensional laminar premixed flames. The concentrations difference 


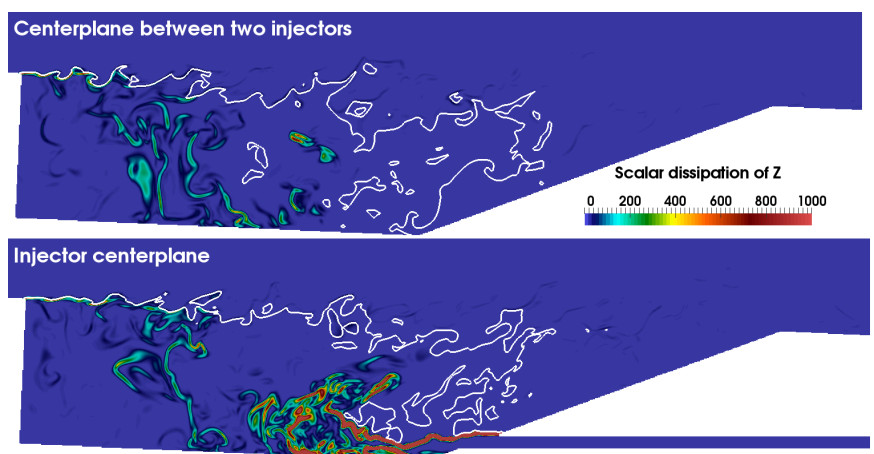

(a) $\chi_{Z}$

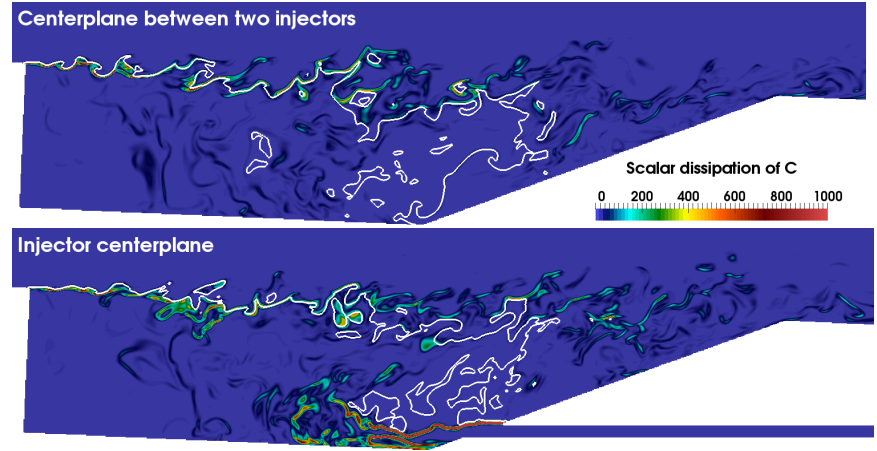

(b) $\chi_{Y_{C}}$
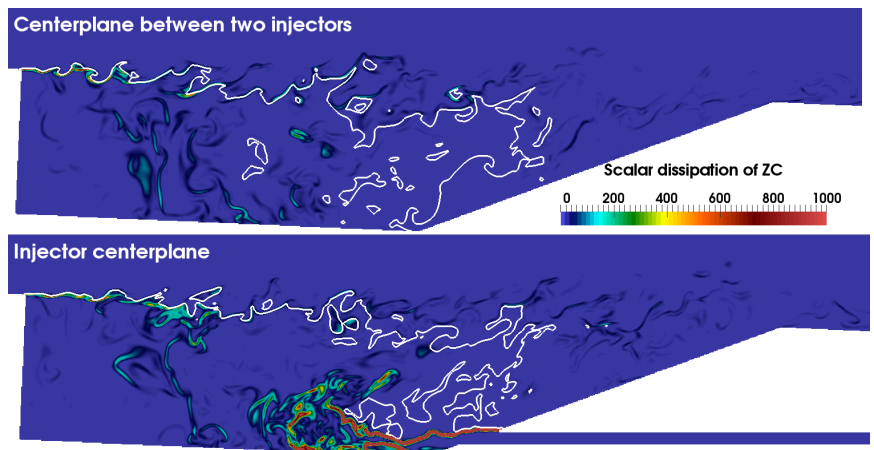

(c) $\chi_{Z C}$

Fig. 30 Instantaneous scalar dissipations in the centerplane between two injectors and in the injector centerplane. The stoichiometric line is displayed in white color. Case RFA1.

between two mixtures around the stoichiometry $\left(\phi_{1}=1.1\right.$ and $\left.\phi_{2}=1.15\right)$ and between two rich mixtures $\left(\phi_{1}=2.1\right.$ and $\left.\phi_{2}=2.15\right), \Delta Y_{k}=\left|Y_{k, 2}-Y_{k, 1}\right|$ with $k=\mathrm{CO}, \mathrm{CO}_{2}$ or $\mathrm{H}_{2} \mathrm{O}$ against the progress variable are displayed in Fig. 34 . $\Delta Y_{k}$ is small at low values of progress variable for all the studied species. But for high values of progress variable $(C>0.9)$, the difference becomes large for 

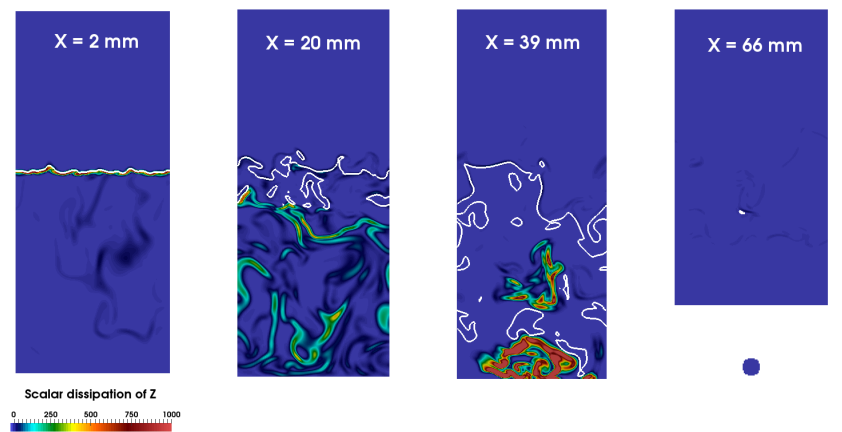

(a) $\chi_{Z}$
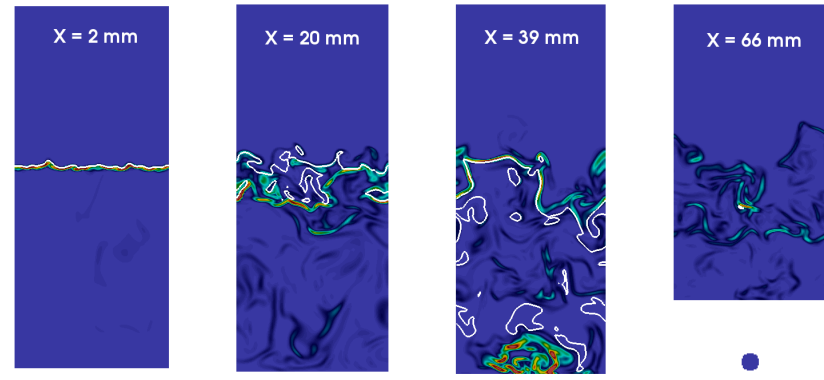

Scalder dissipation of

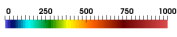

(b) $\chi_{Y_{C}}$
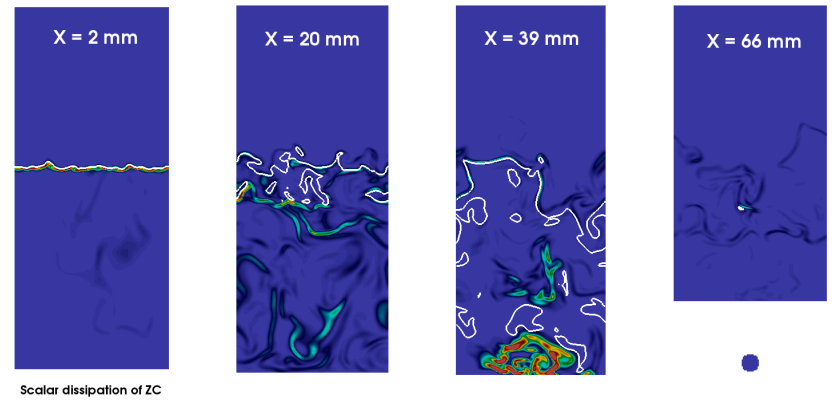

Scalar dissipation of $2 C$

(c) $\chi_{Z C}$

Fig. 31 Instantaneous scalar dissipations in the small recirculation zone $(x=2 \mathrm{~mm})$, the interface between the recirculation zones $(x=20 \mathrm{~mm})$, the large recirculation zone $(x=39$ $\mathrm{mm})$ and the rear of the cavity $(x=66 \mathrm{~mm})$. The stoichiometric line is displayed in white color. Case RFA1. 

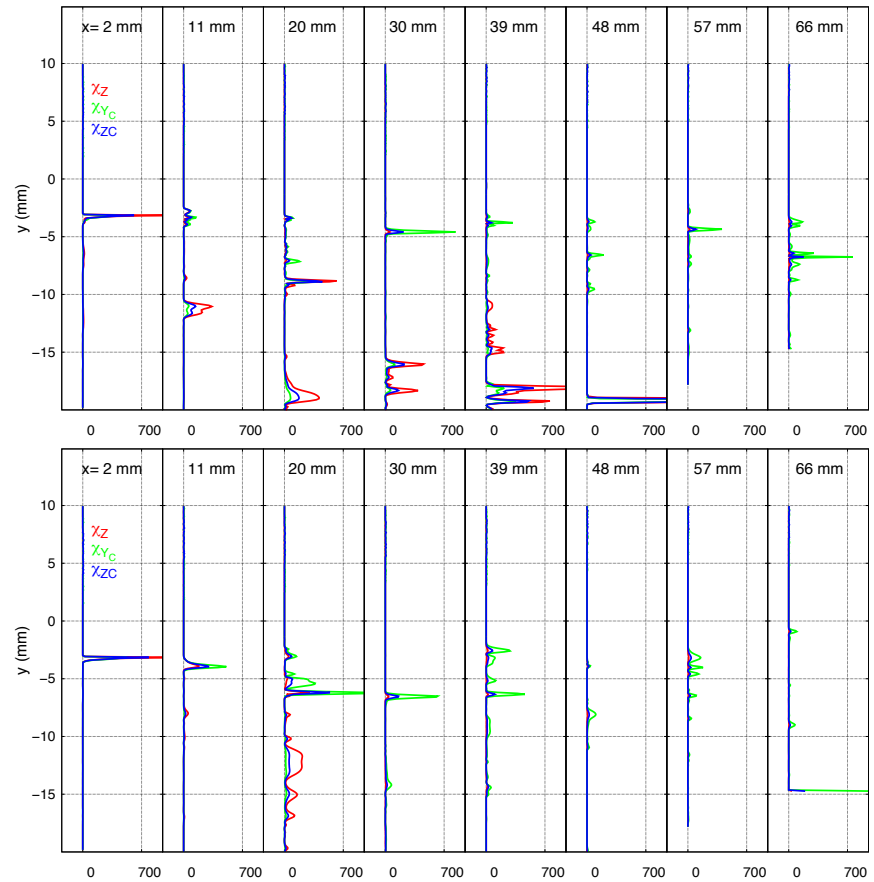

Fig. 32 Instantaneous scalar dissipations profiles in the injector centerplane (top) and in the centerplane between two injectors (bottom). Case RFA1.
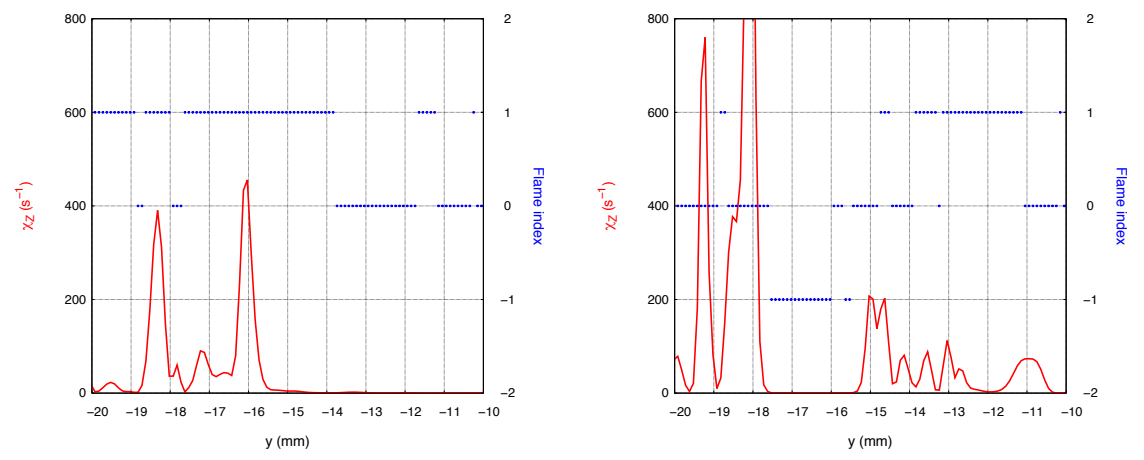

Fig. 33 Instantaneous fields of scalar dissipation $\chi_{Z}$ and flame index in the injector centerplane at $x=30 \mathrm{~mm}$ (left) and $x=39 \mathrm{~mm}$ (right). Case RFA1.

$\mathrm{CO}$ and $\mathrm{CO}_{2}$ for mixtures around the stoichiometry, and stays small for $\mathrm{H}_{2} \mathrm{O}$. For rich mixtures, only the mass fraction of $\mathrm{CO}$ is significantly affected by the change in equivalence ratio. As the mixture fraction in the large recirculation area is around the stoichiometry and the progress variable in this region being higher than 0.9, the large discrepancies found between LES and TAB3 for $\mathrm{CO}$ and $\mathrm{CO}_{2}$ could be explained by these species being more sensitive to 
mixture change. In presence of high $\chi_{Z}$, even for premixed regime, the effect of the local gradient of mixture fraction cannot be neglected with a tabulated approach context. New methods including gradient of mixture fraction in the library construction could help $[27,20]$.
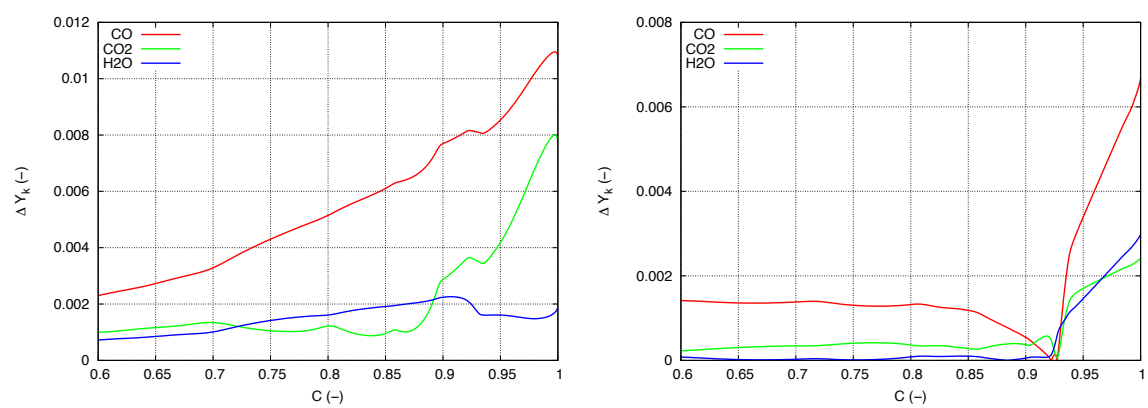

Fig. 34 Sensitivity of $\mathrm{CO}, \mathrm{CO}_{2}$ and $\mathrm{H}_{2} \mathrm{O}$ to the equivalence ratio: $\Delta Y_{k}$ vs. progress variable $C$ for mixtures around the stoichiometry (left) and for rich mixtures (right).

\section{Conclusions}

The large-eddy simulation (LES) of combustion in a cavity-based scramjet has been performed with success with a dynamic approach for subgrid fluxes modeling and a reduced but accurate kinetics. The numerical results are in fair agreement with the experimental measurements available for the AFRL testbench whatever the number of injectors used in the simulation. However, only the simulation of the complete geometry revealed a transverse phenomenon, i.e. the apparition of a M structure of the flame when the exact dimensions and boundary conditions of the cavity are included. In this device, the combustion takes place in region where the flow is largely subsonic and preferentially in the mixing layer over the cavity. Diffusion layers are also present. Highly refined LES has proved to be a reliable tool for gathering information about the dynamics of the combustion in this complex configuration without any need to tune or adjust models.

An a priori tabulated approach for the chemistry was tested based on a collection of one-dimensional premixed low-Mach flames. Good results on temperature and $\mathrm{H}_{2} \mathrm{O}$ fields are found but some discrepancies appeared on $\mathrm{CO}$ and $\mathrm{CO}_{2}$ fields due to the variety of combustion regimes. As expected, shortcomings of the tabulation are present in zones where non-premixed regime is dominant and far from stoichiometry especially in the small recirculation. The impact of strong mixture fraction scalar dissipation in region where combustion is premixed has been evidenced implying that the gradient of mixture fraction should be included in the construction of the look-up table. Never- 
theless, as the pressure in the cavity is relatively uniform and the comparison between LES and TAB3 (built with constant pressure $P=66 \mathrm{kPa}$ ) led to satisfying results for a quantity such as temperature, there is no need to account for the compressibility effect in the tabulation for this scramjet. Tabulation constructed with premixed flamelets including the effects of $Z$ gradients, nonpremixed flamelets, taking into account the dilution by burnt gases in the case of a non adiabatic system, could be then envisaged in the future to model the combustion.

\section{Acknowledgments}

Computations ressources were provided by CRIANN and GENCI (allocations 2017 and 2018-020152).

\section{Compliance with Ethical Standards}

Funding: This study was funded by the French Department of Defense (DGA) (grant number 2015089).

Conflict of Interest: The authors declare that they have no conflict of interest.

\section{References}

1. Babkin, V., Bunev, V., Bolshova, T.: Superadiabatic temperature phenomenon in the combustion process due to a competition between chemical reactions. Combust., Explosion, Shock Waves 51(2), 151-159 (2015)

2. Baurle, R.A.: Hybrid reynolds-averaged / large eddy simulation of a scramjet cavity flameholder. AIAA J. 55(2) (2017)

3. Bioche, K., Vervisch, L., Ribert, G.: Premixed flame-wall interaction in a narrow channel: Impact of wall thermal conductivity and heat losses. J. Fluid Mech. 856, $5-35$ (2018)

4. Bouheraoua, L., Domingo, P., Ribert, G.: Large-eddy simulation of a supersonic lifted jet flame: Analysis of the turbulent flame base. Combust. Flame 179, 199-218 (2017)

5. Candel, S., Schmitt, T., Darabiha, N.: Progress in transcritical combustion: Experimentation, modeling and simulation. In: 23rd ICDERS (2011)

6. Cheng, T., Wehrmeyer, J., Pitz, R., Jarrett, O., Northam, G.: Raman measurement of mixing and finite-rate chemistry in a supersonic hydrogen-air diffusion flame. Combust. Flame 99(1), 157-173 (1994)

7. Domingo, P., Vervisch, L., Veynante, D.: Large-eddy simulation of a lifted methane jet flame in a vitiated coflow. Combust. Flame 152(3), 415-432 (2008)

8. Duboc, B., Ribert, G., Domingo, P.: Description of kerosene / air combustion with hybrid transported-tabulated chemistry. Fuel 233, 146-158 (2018)

9. Duboc, B., Ribert, G., Domingo, P.: Evaluation of chemistry models on methane/air edge flame simulation. Proc. Combust. Inst. 37(2), 1691-1698 (2019)

10. Duboc, B., Ribert, G., Domingo, P.: Hybrid transported-tabulated chemistry for partially premixed combustion. Comput. Fluids 179, 206-227 (2019)

11. Fiorina, B., Gicquel, O., Vervisch, L., Carpentier, S., Darabiha, N.: Approximating the chemical structure of partially premixed and diffusion counterflow flames using FPI flamelet tabulation. Combust. Flame 140, 147-160 (2005) 
12. Gicquel, O., Thévenin, D., Darabiha, N.: Influence of differential diffusion on superequilibrium temperature in turbulent non-premixed hydrogen/air flames. Flow Turbul. Combust. 73, 307-321 (2004)

13. Gonzalez-Juez, E., Kerstein, A., Ranjan, R., Menon, S.: Advances and challenges in modeling high-speed turbulent combustion in propulsion systems. Prog. Energy Combust. Sci. 60, 26-67 (2017)

14. Guven, U., Ribert, G.: Large-eddy simulation of supersonic hydrogen/oxygen combustion: application to rocketlike igniter. J. Propul. Power 34(2), 291-307 (2018)

15. Guven, U., Ribert, G.: Impact of non-ideal transport modeling on supercritical flow simulation. Proc. Combust. Inst. 37(3), 3255 - 3262 (2019)

16. Hassan, E., Peterson, D., Walters, D., Luke, E.: Dynamic hybrid reynolds-averaged navier-stokes/large-eddy simulation of a supersonic cavity: Chemistry effects. J. Propul. Power 35(1), 201-212 (2019)

17. Hassan, E., Peterson, D., Walters, K., Luke, E.: Dynamic hybrid reynolds-averaged navier stokes/large-eddy simulation of a supersonic cavity. J. Propul. Power 32(6), 1343-1352 (2016)

18. Kopp, M., Donato, N., Petersen, E., Metcalfe, W., Burke, S., Curran, H.: Oxidation of ethylene-air mixtures at elevated pressures, part 1: Experimental results. J. Propul. Power 30(3), 790 - 798 (2014)

19. Liu, F., Guo, H., Smallwood, G., Gülder, O.: Numerical study of the superadiabatic flame temperature phenomenon in hydrocarbon premixed flames. Proc. Combust. Inst. 29, 1543-1550 (2002)

20. Lodier, G., Vervisch, L., Moureau, V., Domingo, P.: Composition-space premixed flamelet solution with differential diffusion for in situ flamelet-generated manifolds. Combust. Flame 158, 2009-2016 (2011)

21. Luo, Z., Yoo, C.S., Richardson, E.S., Chen, J.H., Law, C.K., Lu, T.F.: Chemical explosive mode analysis for a turbulent lifted ethylene jet flame in highly-heated coflow. Combust. Flame 159(1), 265-274 (2012)

22. Lutz, A., Kee, R., Miller, J.: SENKIN - A Fortran program for predicting homogeneous gas phase chemical kinetics with sensitivity analysis. Tech. rep., Report No. SAND878240 UC-4, Sandia Lab. (1990)

23. Lutz, A., Rupley, F., Kee, R., Reynolds, W., Meeks, E.: EQUIL : A CHEMKIN implementation of STANJAN for computing chemical equilibria. Tech. rep., Reaction Design (1998)

24. Meeks, E., Kee, R., Dandy, D., Coltrin, M.: Computational simulation of diamond chemical vapor deposition in premixed $\mathrm{C}_{2} \mathrm{H}_{2} / \mathrm{O}_{2} / \mathrm{H}_{2}$ and $\mathrm{CH}_{4} / \mathrm{O}_{2}$ strained flames. Combust. Flame 92, 144-160 (1993)

25. Metcalfe, W., Burke, S., Ahmed, S., Curran, H.: A hierarchical and comparative kinetic modeling study of $\mathrm{C}_{1}-\mathrm{C}_{2}$ hydrocarbon and oxygenated fuels. Int. J. Chem. Kinet. 45(10), $638-675$ (2013)

26. Najafi-Yazdi, A., Cuenot, B., Mongeau, L.: Systematic definition of progress variables and intrinsically low-dimensional, flamelet generated manifolds for chemistry tabulation. Combust. Flame 159(3), 1197-1204 (2012)

27. Nguyen, P.D., Vervisch, L., Subramanian, V., Domingo, P.: Multidimensional flameletgenerated manifolds for partially premixed combustion. Combust. Flame 157, 43-61 (2010)

28. Niu, Y.S., Vervisch, L., Tao, P.D.: An optimization-based approach to detailed chemistry tabulation: Automated progress variable definition. Combust. Flame 160(4), 776-785 (2013)

29. van Oijen, J., Donini, A., Bastiaans, R., ten Thije Boonkkamp, J., de Goey, L.: Stateof-the-art in premixed combustion modeling using flamelet generated manifolds. Prog. Energy Combust. Sci. 57, 30-74 (2016)

30. Pons, L., Darabiha, N., Candel, S., Ribert, G., Yang, V.: Mass transfer and combustion in transcritical non-premixed counterflows. Combust. Theory Model. 13, 57-81 (2008)

31. Pope, S.: Small scales, many species and the manifold challenges of turbulent combustion. Proc. Combust. Inst. 34(1), 1-31 (2013)

32. Ribert, G., Vervisch, L., Domingo, P., Niu, Y.S.: Hybrid transported-tabulated strategy to downsize detailed chemistry for numerical simulation of premixed flames. Flow Turbul. Combust. 92, 175-200 (2014) 
33. Ribert, G., Zong, N., Yang, V., Pons, L., Darabiha, N., Candel, S.: Counterflow diffusion flames of general fluids: oxygen/hydrogen mixtures. Combust. Flame 154, 319-330 (2008)

34. Ruan, J.L., Domingo, P., Ribert, G.: Analysis of combustion modes in a cavity based scramjet. Combust. Flame 251, 238-251 (2020)

35. Saghafian, A., Shunn, L., Philips, D.A., Ham, F.: Large eddy simulations of the hifire scramjet using a compressible flamelet/progress variable approach. Proc. Combust. Inst. 35, 2163-2172 (2015)

36. Saghafian, A., Terrapon, V.E., Pitsch, H.: An an efficient flamelet-based combustion model for compressible flows. Combust. Flame 162, 652-667 (2015)

37. Tuttle, S.G., Carter, C.D., Hsu, K.Y.: Particle image velocimetry in an isothermal and exothermic high-speed cavity. J. Propul. Power 30(13) (2014)

38. Wang, K., Ribert, G., Domingo, P., Vervisch, L.: Self-similar behavior and chemistry tabulation of burnt-gas diluted premixed flamelets including heat-loss. Combust. Theory Model. 14, 541-570 (2010) 


\section{A Progress variable}

The construction of progress variable needs to satisfy two conditions:

(I) the progress variable $C$ must be a monotonically increasing function of the physical coordinate $X$

(II) all tabulated quantities must be injective functions of the progress variable $C$.

The verification is done for $\phi \in[0.4,5]$ in Fig. 35 for (I) and in Fig. 36 for (II).
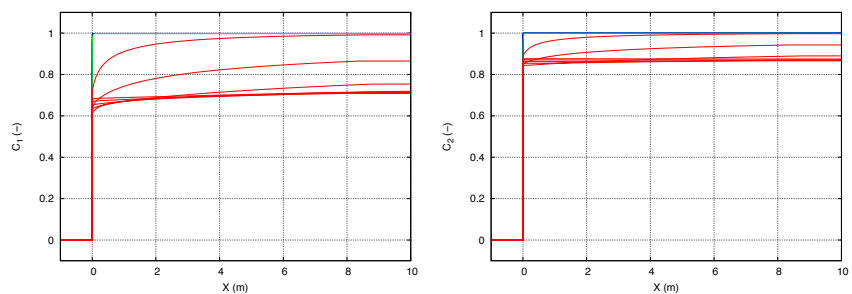

Fig. 35 Progress variables $C_{1}$ (left) and $C_{2}$ (right) in physical space at equivalence ratio $\phi \in[0.4,5]$ : blue, green and red colors correspond respectively to lean, stoichiometric and rich mixtures.
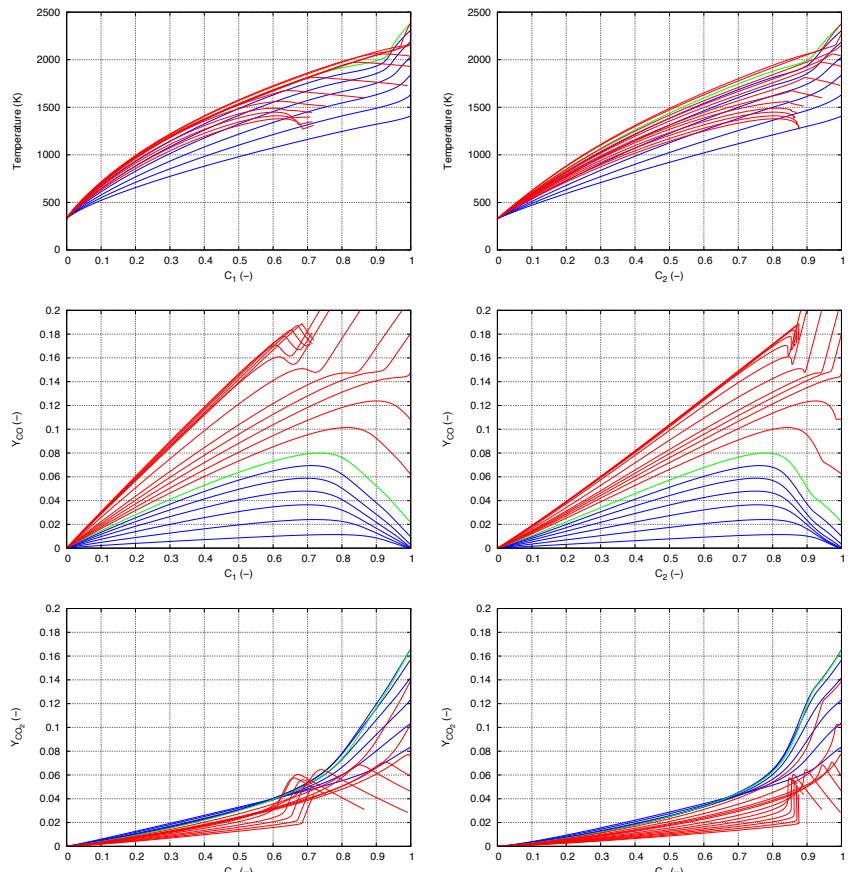

Fig. 36 Tabulated temperature and major combustion product mass fractions against progress variables $C_{1}$ (left) and $C_{2}$ (right) at equivalence ratio $\phi \in[0.4,5]$ : blue, green and red colors correspond respectively to lean, stoichiometric and rich mixtures. 